\title{
JUMP DEGREES OF TORSION-FREE ABELIAN GROUPS
}

\author{
BROOKE M. ANDERSEN, ASHER M. KACH, ALEXANDER G. MELNIKOV, \\ AND REED SOLOMON
}

\begin{abstract}
We show, for each computable ordinal $\alpha$ and degree $\mathbf{a}>\mathbf{0}^{(\alpha)}$, the existence of a torsion-free abelian group with proper $\alpha^{t h}$ jump degree $\mathbf{a}$.
\end{abstract}

\section{INTRODUCTION}

An important topic of effective algebra is concerned with determining the computational strength of an algebraic structure. For an isomorphism type $\mathcal{A}$, it is natural to define the computational strength of $\mathcal{A}$ (the degree of $\mathcal{A}$ ) to be the least degree (if it exists) in its degree spectrum

$$
\operatorname{Deg} \operatorname{Spec}(\mathcal{A})=\{\mathbf{d}: \mathcal{A} \text { is } \mathbf{d} \text {-computable }\} .
$$

We recall an infinite algebraic structure $\mathcal{A}$ is $\mathbf{d}$-computable if its universe can be identified with the natural numbers $\omega$ in such a way that the atomic diagram of $\mathcal{A}$ becomes d-computable.

Often, the degree spectrum fails to capture the computational strength of $\mathcal{A}$ as in many cases it has no least element (for example, see [18]). As a result, there has been a line of study into the jump degrees of isomorphism types.

Definition 1.1. If $\mathcal{A}$ is any countable structure, $\alpha$ is any computable ordinal, and $\mathbf{a} \geq \mathbf{0}^{(\alpha)}$ is any degree, then $\mathcal{A}$ has $\alpha^{\text {th }}$ jump degree $\mathbf{a}$ if the set

$$
\left\{\mathbf{d}^{(\alpha)}: \mathbf{d} \in \operatorname{DegSpec}(\mathcal{A})\right\}
$$

has a as its least element. In this case, the structure $\mathcal{A}$ is said to have $\alpha^{\text {th }}$ jump degree.

A structure $\mathcal{A}$ has proper $\alpha^{\text {th }}$ jump degree a if $\mathcal{A}$ has $\alpha^{\text {th }}$ jump degree a but not $\beta^{\text {th }}$ jump degree for any $\beta<\alpha$. In this case, the structure $\mathcal{A}$ is said to have proper $\alpha^{\text {th }}$ jump degree.

For any computable ordinal $\alpha$, it is well-known that an arbitrary structure may not have $\alpha^{\text {th }}$ jump degree (for example, see [6]). The existence or nonexistence of a structure with proper $\alpha^{\text {th }}$ jump degree $\mathbf{a}$ for $\mathbf{a} \geq \mathbf{0}^{(\alpha)}$ depends heavily on the class of algebraic structures considered. Within the context of linear orders, if an order type has a degree, it must be $\mathbf{0}$; if an order type has first jump degree, it must be $\mathbf{0}^{\prime}$; and yet for each computable ordinal $\alpha \geq 2$ and degree $\mathbf{a} \geq \mathbf{0}^{(\alpha)}$, there is a linear order having proper $\alpha^{\text {th }}$ jump degree a (see [6], culminating results in [1], [13], [15] and [18]). Within the context of Boolean algebras, if a Boolean algebra has $n^{\text {th }}$

Date: June 30, 2011.

Key words and phrases. torsion-free abelian group, jump degree.

The second author was partially supported by the Marsden Fund of New Zealand via a PostDoctoral Fellowship. 
jump degree (for any $n \in \omega$ ), it must be $\mathbf{0}^{(n)}$; yet for each $\mathbf{a} \geq \mathbf{0}^{(\omega)}$, there is a Boolean algebra with proper $\omega^{\text {th }}$ jump degree a (see [14]).

The subject of this paper is the existence of jump degrees of torsion-free abelian groups. For $\alpha \in\{0,1,2\}$, it is known that every possible proper $\alpha^{\text {th }}$ jump degree is realized.

Theorem 1.2 (Downey [5]; Downey and Jockusch [5]). For every degree $\mathbf{a} \geq \mathbf{0}$, there is a (rank one) torsion-free abelian group having degree $\mathbf{a}$.

For every degree $\mathbf{b} \geq \mathbf{0}^{\prime}$, there is a (rank one) torsion-free abelian group having proper first jump degree $\mathbf{b}$.

Indeed, every finite rank torsion-free abelian group (see Definition 2.2) has first jump degree as a consequence of a difficult computability theoretic theorem of Coles, Downey, and Slaman [3]. (See Melnikov [17] for a complete discussion.)

In contrast, not every infinite rank torsion-free abelian group has first jump degree as a consequence of the following theorem of Melnikov. Recall that a nonzero degree $\mathbf{a}$ is low if $\mathbf{a}^{\prime}=\mathbf{0}^{\prime}$ and nonlow otherwise.

Theorem 1.3 (Melnikov [17]). There is a torsion-free abelian group $\mathcal{G}$ such that $\operatorname{DegSpec}(\mathcal{G})=\{\mathbf{a}: \mathbf{a}$ is nonlow $\}$. Consequently, there is a torsion-free abelian group with proper second jump degree $\mathbf{0}^{\prime \prime}$.

As a consequence of work by Ash, Jockusch, and Knight (see [1]), two observations of Melnikov (see Theorem 3 and Proposition 10 of [17]) have implications about proper second jump degrees and proper third jump degrees.

Theorem 1.4. For every degree $\mathbf{a}>\mathbf{0}^{\prime \prime}$, there is a torsion-free abelian group having proper second jump degree $\mathbf{a}$. For every degree $\mathbf{b}>\mathbf{0}^{\prime \prime \prime}$, there is a torsion-free abelian group having proper third jump degree $\mathbf{b}$.

The main purpose of this paper is to generalize Theorem 1.4 to an arbitrary computable ordinal $\alpha$.

Theorem 1.5. For every computable ordinal $\alpha$ and degree $\mathbf{a}>\mathbf{0}^{(\alpha)}$, there is a torsion-free abelian group having proper $\alpha^{\text {th }}$ jump degree $\mathbf{a}$.

Unfortunately, our techniques do not allow us to produce examples with $\mathbf{a}=\mathbf{0}^{(\alpha)}$.

Question 1.6. For each computable ordinal $\alpha \geq 3$, is there a torsion-free abelian group having proper $\alpha^{\text {th }}$ jump degree $\mathbf{0}^{(\alpha)}$ ?

Fixing $\alpha$, we prove Theorem 1.5 by coding sets $S \subseteq \omega$ into groups $\mathcal{G}_{S}$ in such a way that $\mathcal{G}_{S}$ is $X$-computable if and only if $S \in \Sigma_{\alpha}^{0}(X)$. The coding method is based on techniques in Fuchs (see Section XIII, Chapter 88 and Chapter 89, of [11]) and Hjorth (see [12]). In particular, given torsion-free abelian groups $\mathcal{A}$ and $\mathcal{B}$ of a certain type and elements $a \in A$ and $b \in B$, Fuchs adds elements of the form $p^{-n}(a+b)$ for $n \in \omega$ to $\mathcal{A} \oplus \mathcal{B}$ to build an indecomposable group containing $\mathcal{A} \oplus \mathcal{B}$. Hjorth uses this idea to encode certain labeled graphs into countable torsion-free abelian groups. Hjorth's ideas were then used by Downey and Montalbán to show the isomorphism problem for torsion-free abelian groups is analytic complete (see [7]) and further studied by Fokina, Knight, Maher, Melnikov, and Quinn (see [8]). We refer the reader to these papers for additional background, though we do not assume knowledge of them in our presentation. Our notation and terminology will follow Fuchs (see [10] and [11]) rather than Hjorth. 
Section 2 discusses background, notation, and conventions, though we also refer the reader to [10] and [11] for classical background on torsion-free abelian groups and to [2] for background on effective algebra. Section 3 describes the encoding of sets $S \subseteq \omega$ into groups $\mathcal{G}_{S}$ (this encoding depends on $\alpha$ ). Theorem 1.5 is demonstrated in Section 4.

\section{Background, Notation, and Conventions}

In this section, we review basic terminology and results relevant to torsionfree abelian groups. We also introduce some classical notation and adopt some conventions that will simplify the exposition.

Definition 2.1. An abelian group $\mathcal{G}=(G:+, 0)$ is torsion-free if

$$
(\forall x \in G)(\forall n \in \omega)[x \neq 0 \wedge n \neq 0 \Longrightarrow n x \neq 0]
$$

where $n x$ denotes $\underbrace{x+\cdots+x}_{n \text { times }}$.

Definition 2.2. If $\mathcal{G}$ is a torsion-free abelian group, a (finite or infinite) set of nonzero elements $\left\{g_{i}\right\}_{i \in I} \subset G$ is linearly independent if $\alpha_{1} g_{i_{1}}+\cdots+\alpha_{k} g_{i_{k}}=0$ has no solution with $\alpha_{i} \in \mathbb{Z}$ for each $i,\left\{i_{1}, \ldots, i_{k}\right\} \subseteq I$, and $\alpha_{i} \neq 0$ for some $i$. A basis for $\mathcal{G}$ is a maximal linearly independent set and the rank of $\mathcal{G}$ is the cardinality of a basis.

The groups $\mathcal{G}$ constructed for Theorem 1.5 will have countably infinite rank. The key coding mechanism will be the existence or nonexistence of elements divisible by arbitrarily high powers of a prime.

Definition 2.3. If $\mathcal{G}$ is a torsion-free abelian group and $x \in G$, we write $p^{\infty} \mid x$ and say $p$ infinitely divides $x$ if $(\forall k \in \omega)(\exists y \in G)\left[x=p^{k} y\right]$, i.e., if $p$ divides $x$ arbitrarily many times. We say $p$ finitely divides $x$ and write $p^{\infty} \not x$ otherwise.

Definition 2.4. A subgroup $\mathcal{G}$ of an abelian group $\mathcal{H}$ is pure if for every $n \in \mathbb{Z}$ and every $b \in G$ :

$$
\text { If } \mathcal{H} \models n \mid b \text {, then } \mathcal{G} \models n \mid b \text {. }
$$

In other words, if an integer $n$ divides an element $b \in G$ within $\mathcal{H}$, then $n$ divides $b$ within $\mathcal{G}$.

We note that if $\mathcal{G}$ is torsion-free, then $x=p^{k} y$ can have at most one solution $y$ for any integer $k$.

Remark 2.5. Within any presentation of $\mathcal{G}$, the set $\left\{x \in G: p^{\infty} \mid x\right\}$ of elements infinitely divisible by $p$ is $\Pi_{2}^{c}(\mathcal{G})$. Indeed, this set is a subgroup of $\mathcal{G}$ under the group operation (which we use without further mention).

Definition 2.6. A torsion-free abelian group is divisible if it is the additive group of a $\mathbb{Q}$-vector space (or equivalently, if $x=n y$ has a solution $y \in G$ for every $x \in G$ and $n \geq 1)$.

If $\mathcal{G}$ is a torsion-free abelian group, its divisible closure (denoted $D(\mathcal{G})$ ) is the divisible torsion-free abelian group of the least rank containing $\mathcal{G}$. (This least rank is the rank of $\mathcal{G}$.) 
Thus, the countable divisible torsion-free abelian groups are the groups $\mathbb{Q}^{n}$ (for $n \in \omega$ ) and $\mathbb{Q}^{\omega}$, and the divisible closure of $\mathbb{Z}$ is $\mathbb{Q}$. Classically, the divisible closure $D(\mathcal{G})$ exists, is unique, and contains $\mathcal{G}$ as a subgroup. In terms of effective algebra, Smith (see [20]) proved that every computable torsion-free abelian group has a computable divisible closure and that there is a uniform procedure for passing from $\mathcal{G}$ to $D(\mathcal{G}){ }^{1}$ However, in general the divisible closure is not effectively unique (i.e., unique up to computable isomorphism) and the canonical image of $\mathcal{G}$ in $D(\mathcal{G})$ is computably enumerable but not necessarily computable (see [9] and [21] for a complete discussion of these issues). Therefore, when we consider a particular copy $\mathcal{G}$ of a torsion-free abelian group, we use $D(\mathcal{G})$ to denote the canonical divisible closure as in [20]. Thus, we have a uniform way to pass from any given copy of $\mathcal{G}$ to a copy of $D(\mathcal{G})$.

In our construction, we will use a more limited notion of closure under divisibility by certain primes.

Definition 2.7. If $p \in \omega$ is prime and $\mathcal{G}$ is a torsion-free abelian group, define the $p$-closure of $\mathcal{G}\left(\right.$ denoted $[\mathcal{G}]_{p}$ ) to be the smallest subgroup $\mathcal{H}$ of $D(\mathcal{G})$ containing $\mathcal{G}$ having the property $(\forall g \in G)\left[p^{\infty} \mid g\right]$.

More generally, if $P$ is a set of prime numbers and $\mathcal{G}$ is a torsion-free abelian group, define the $P$-closure of $\mathcal{G}$ (denoted $\left.[\mathcal{G}]_{P}\right)$ to be the smallest subgroup $\mathcal{H}$ of $D(\mathcal{G})$ containing $\mathcal{G}$ having the property $(\forall g \in G)(\forall p \in P)\left[p^{\infty} \mid g\right]$. We often write $[\mathcal{G}]_{p_{0}, p_{1}}$ for $[\mathcal{G}]_{P}$ with $P=\left\{p_{0}, p_{1}\right\}$ and $[\mathcal{G}]_{P, q}$ for $[\mathcal{G}]_{P \cup\{q\}}$.

If $\mathcal{G}$ is any torsion-free abelian group and $P$ is any set of prime numbers, we say that $\mathcal{G}$ is $P$-closed if $\mathcal{G} \cong[\mathcal{G}]_{P}$.

The following lemma says that if $\mathcal{G}$ is $P$-closed, then the result of closing $\mathcal{G}$ under additional primes will still be $P$-closed. In particular, we can view the prime closure $[\mathcal{G}]_{P}$ as the result of closing $\mathcal{G}$ under each of the individual primes in $P$ in any order.

Lemma 2.8. If $\mathcal{G}$ is a torsion free abelian group, $P$ is a set of primes and $q$ is prime not in $P$, then $\left[[\mathcal{G}]_{P}\right]_{q} \cong[\mathcal{G}]_{P, q}$.

Proof. Since $\left[[\mathcal{G}]_{P}\right]_{q}$ is clearly a subgroup of $[\mathcal{G}]_{P, q}$ and since element of $\left[[\mathcal{G}]_{P}\right]_{q}$ is infinitely divisible by $q$, it suffices to show that each element of $\left[[\mathcal{G}]_{P}\right]_{q}$ is infinitely divisible by each prime $p \in P$. Fix $p \in P$ and $g \in\left[[\mathcal{G}]_{P}\right]_{q}$. We need to find $h \in\left[[\mathcal{G}]_{P}\right]_{q}$ such that $p h=g$. By the definition of $\left[[\mathcal{G}]_{P}\right]_{q}$, there is a $k \geq 0$ such that $q^{k} g=\widehat{h} \in[\mathcal{G}]_{P}$. Let $\widehat{g} \in[\mathcal{G}]_{P}$ be such that $p \widehat{g}=\widehat{h}$ and let $h \in\left[[\mathcal{G}]_{P}\right]_{q}$ be such that $q^{k} h=\widehat{g}$. Then

$$
q^{k}(p h)=p\left(q^{k} h\right)=p \widehat{g}=\widehat{h}=q^{k} g
$$

Since $\mathcal{G}$ is torsion free, $q^{k}(p h)=q^{k} g$ implies $p h=g$ as required.

By an obvious variation of the construction in [20], there is an effective way to pass from $\mathcal{G}$ to a copy of $[\mathcal{G}]_{P}$ which is uniform in both $\mathcal{G}$ and $P$. As above, the closure operation sending $\mathcal{G}$ to $[\mathcal{G}]_{P}$ is not necessarily effectively unique so we fix this uniform procedure to define a particular copy of $[\mathcal{G}]_{P}$ given a particular copy of $\mathcal{G}$.

Convention 2.9. We will write statements such as $\left([\mathbb{Z}]_{\rho_{1}, P} \backslash[\mathbb{Z}]_{P}\right) \cap[\mathbb{Z}]_{\rho_{2}, \rho_{3}, P}=\emptyset$. Such statements are intended to apply within a fixed (one-dimensional) copy of $\mathbb{Q}$,

\footnotetext{
${ }^{1}$ One forms $D(\mathcal{G})$ from pairs $\langle g, n\rangle$ with $g \in \mathcal{G}$ and $n \geq 1$ modulo the computable equivalence relation $\langle g, n\rangle \sim\langle h, m\rangle$ if and only if $m g=n h$.
} 
where $\mathbb{Z} \leqq \mathbb{Q}$ is fixed as well. In particular, the indicated prime closures of $\mathbb{Z}$ should all be seen as being taken within a fixed copy of $\mathbb{Q}$.

Often, we will write elements of the form $\frac{x+y}{p}$ as $\frac{x}{p}+\frac{y}{p}$ even though $\frac{x}{p}$ and $\frac{y}{p}$ may not exist within the group. We justify this by passing to the divisible closure of the group and considering the canonical image of the group within its divisible closure. Thus, $\frac{x+y}{p}=\frac{x}{p}+\frac{y}{p}$ in $D(\mathcal{G})$ even though $\frac{x}{p}$ and $\frac{y}{p}$ may not be in the image of $\mathcal{G}$. However, when we speak of an element $z \in \mathcal{G}$ being infinitely divisible by a prime $p$, we mean its image in the divisible closure is infinitely divisible by the prime $p$ and this divisibility is witnessed by elements that are the images of elements of $\mathcal{G}$.

Definition 2.10. A rooted torsion-free abelian group $\mathcal{G}$ is a torsion-free abelian group with a distinguished element (termed the root of $\mathcal{G}$ ).

We use rooted torsion-free abelian groups to help build our groups inductively. When we consider isomorphisms, we always consider group isomorphisms with no assumption that roots are preserved. That is, the root is only used as a tool in the inductive definitions and is not a formal part of the algebraic structure.

Definition 2.11. Let $\mathcal{G}$ be a torsion-free abelian group and $\left\{d_{i}\right\}_{i \in I} \subseteq D(\mathcal{G})$ be a subset of its divisible closure. We define the extension of $\mathcal{G}$ by $\left\{d_{i}\right\}_{i \in I}$, denoted

$$
\left\langle\mathcal{G} ; d_{i}: i \in I\right\rangle,
$$

to be the smallest subgroup of $D(\mathcal{G})$ containing $\mathcal{G}$ and $d_{i}$ for $i \in I$.

Note that if $\mathcal{G}$ is computable and $\left\{d_{i}\right\}_{i \in I}$ is a computable set of elements of $D(\mathcal{G})$ (indeed, computably enumerable suffices), then the subgroup $\left\langle\mathcal{G} ; d_{i}: i \in I\right\rangle$ is computably enumerable in $D(\mathcal{G})$. Since there is a uniform procedure to produce a computable copy of any computably enumerable subgroup of $D(\mathcal{G})$ (by letting $n$ denote the $n$-th element enumerated into the subgroup and defining the group operations accordingly) we have a uniform procedure to pass from $\mathcal{G}$ to $\left\langle\mathcal{G} ; d_{i}: i \in I\right\rangle$.

We continue by introducing some (important) conventions that will be used throughout the paper without further mention.

Convention 2.12. If $\beta$ is any nonzero ordinal, when we write $\beta=\delta+i$ or $\beta=$ $\delta+2 \ell+i$ for some $i \in \omega$, we require $\delta$ to be either zero or a limit ordinal (allowing zero only if $\beta<\omega$ ) and $\ell$ to be a nonnegative integer.

If $i$ is even, we say the ordinal $\beta$ is even; if $i$ is odd, we say the ordinal $\beta$ is odd.

When at limit ordinals, it will be necessary to approximate the ordinal effectively from below. We therefore fix a computable ordinal $\lambda$ and increasing cofinal sequences for ordinals less than $\lambda$.

Definition 2.13. Fix a computable ordinal $\lambda$.

Fix a computable function $f: \lambda \times \omega \rightarrow \lambda$ such that $f(\alpha+1, n)=\alpha$ for all successor ordinals $\alpha+1 \in \lambda$ and $n \in \omega$, and such that $\{f(\alpha, n)\}_{n \in \omega}$ is a sequence of increasing odd ordinals (greater than one) with $\alpha=\cup_{n \in \omega} f(\alpha, n)$ for all limit ordinals $\alpha \in \lambda$.

We denote $f(\alpha, n)$ by $f_{\alpha}(n)$.

\section{The Group $\mathcal{G}_{S}$ (For Successor Ordinals $\alpha$ )}

Fixing a computable successor ordinal $\alpha$ below $\lambda$, the group $\mathcal{G}_{S}$ will be a direct sum of rooted torsion-free abelian groups $\mathcal{G}_{S}(n)$ coding whether $n$ is or is not in $S$. It 
will be useful to have a plethora of disjoint sets of primes. We therefore partition the prime numbers into uniformly computable sets $P=\left\{p_{\beta}\right\}_{\beta \in \alpha+1}, Q=\left\{q_{\beta}\right\}_{\beta \in \alpha+1}$, $U=\left\{u_{\beta, k}\right\}_{\beta \in \alpha+1, k \in \omega}, V=\left\{v_{\beta, k}\right\}_{\beta \in \alpha+1, k \in \omega}, D=\left\{d_{n}\right\}_{n \in \omega}$, and $E=\left\{e_{n}\right\}_{n \in \omega},$.

More specifically, the isomorphism type of $\mathcal{G}_{S}(n)$ will be either $\left[\mathcal{G}\left(\Sigma_{\alpha}^{0}\right)\right]_{d_{n}}$ or $\left[\mathcal{G}\left(\Pi_{\alpha}^{0}\right)\right]_{d_{n}}$, or $\left[\mathcal{H}\left(\Sigma_{\alpha}^{0}\right)\right]_{d_{n}}$ or $\left[\mathcal{H}\left(\Pi_{\alpha}^{0}\right)\right]_{d_{n}}$ (all described later) depending on whether $\alpha$ is even or odd (deciding $\mathcal{G}$ versus $\mathcal{H}$ ) and whether $n$ is in $S$ (deciding $\Sigma$ versus $\Pi$ ). The group $\mathcal{G}_{S}(n)$ will be $X$-computable (uniformly in $n$ ) if $S \in \Sigma_{\alpha}^{0}(X)$. Conversely, there will be an effective enumeration $\left\{\Upsilon_{n}\right\}_{n \in \omega}$ of computable infinitary $\Sigma_{\alpha}^{c}$ sentences such that $\mathcal{G}_{S} \models \Upsilon_{n}$ if and only if $n \in S .{ }^{2}$ Thus, the group $\mathcal{G}_{S}$ will be $X$-computable if and only if $S \in \Sigma_{\alpha}^{0}(X)$.

The definition of the rooted torsion-free abelian groups $\mathcal{G}\left(\Sigma_{\alpha}^{0}\right), \mathcal{G}\left(\Pi_{\alpha}^{0}\right), \mathcal{H}\left(\Sigma_{\alpha}^{0}\right)$, and $\mathcal{H}\left(\Pi_{\alpha}^{0}\right)$ is done by recursion. Unfortunately, the recursion is not straightforward for technical reasons within the algebra (discussed in Remark 3.2). Indeed, we introduce additional rooted torsion-free abelian groups $\mathcal{G}\left(\Sigma_{\alpha}^{0}(m)\right)$ for $m \in \omega$ if $\alpha$ is an even ordinal.

We define some of these groups pictorially in Section 3.1. The hope is these examples provide enough intuition to the reader so that the formal definition of $\mathcal{G}_{S}$ (and all the auxiliary groups) is not (too) painful.

3.1. Defining $\mathcal{G}\left(\Sigma_{\beta}^{0}\right), \mathcal{G}\left(\Sigma_{\beta}^{0}(m)\right)$, and $\mathcal{G}\left(\Pi_{\beta}^{0}\right)$ Pictorially. For each successor ordinal $\beta \geq 3$, we give a pictorial description of the groups $\mathcal{G}\left(\Sigma_{\beta}^{0}\right), \mathcal{G}\left(\Sigma_{\beta}^{0}(m)\right)$, and $\mathcal{G}\left(\Pi_{\beta}^{0}\right)$. The recursion starts with $\mathcal{G}\left(\Sigma_{2}^{0}(m)\right)$ as $\mathbb{Z}$ with root $r=p_{1}^{m}$ and $\mathcal{G}\left(\Pi_{2}^{0}\right)$ as $[\mathbb{Z}]_{p_{1}}$ with root $r=1$. The recursion continues as illustrated in Figure 1 and Figure 2.

Within these figures, the recursively defined rooted torsion-free abelian groups are denoted with triangles (the text inside specifies which recursively defined group), with the root denoted by a circle at the top. A line segment connecting two roots and with a label $p$ denotes the sum of the roots is infinitely divisible by $p$. Brackets around a recursively defined rooted group with a label $p$ denotes the $p$-closure of the recursively defined rooted group. A prime $p$ next to a root $r$ denotes $r$ is infinitely divisible by $p$.

3.2. Defining $\mathcal{G}_{S}$ Formally. Having pictorially described the groups $\mathcal{G}\left(\Sigma_{\beta}^{0}\right), \mathcal{G}\left(\Sigma_{\beta}^{0}(m)\right)$, and $\mathcal{G}\left(\Pi_{\beta}^{0}\right)$, we formalize the definition of $\mathcal{G}_{S}$. Of course, doing so requires formalizing the definition of all the auxiliary groups.

Definition 3.1. For each ordinal $\beta$ with $1<\beta \leq \alpha$, define rooted torsion-free abelian groups $\mathcal{G}\left(\Sigma_{\beta}^{0}\right)$ and $\mathcal{G}\left(\Pi_{\beta}^{0}\right)$ (if $\beta$ is odd) or $\mathcal{G}\left(\Sigma_{\beta}^{0}(m)\right)$ for $m \in \omega$ and $\mathcal{G}\left(\Pi_{\beta}^{0}\right)$ (if $\beta$ is even) by recursion as follows.

- For $\beta=2$, define $\mathcal{G}\left(\Sigma_{\beta}^{0}(m)\right)$ to be the group $\mathbb{Z}$ with root $r=p_{1}^{m}$ and define $\mathcal{G}\left(\Pi_{\beta}^{0}\right)$ to be the group $[\mathbb{Z}]_{p_{1}}$ with root $r=1$.

- For odd $\beta=\delta+2 \ell+1 \geq 3$, define $\mathcal{G}\left(\Sigma_{\beta}^{0}\right)$ to be the group

$\left\langle[\mathbb{Z}]_{p_{\beta}} \oplus \bigoplus_{k \in \omega} \bigoplus_{m \in \omega} \mathcal{G}\left(\Sigma_{\beta-1}^{0}(m)\right) \oplus \bigoplus_{k \in \omega} \mathcal{G}\left(\Pi_{\beta-1}^{0}\right) ; q_{\beta}^{-t}\left(r+r_{k}\right), q_{\beta}^{-t}\left(r+r_{k, m}\right): k, m, t \in \omega\right\rangle$,

\footnotetext{
${ }^{2}$ We refer the reader to [2], for example, for definitions and background on computable infinitary formulas.
} 

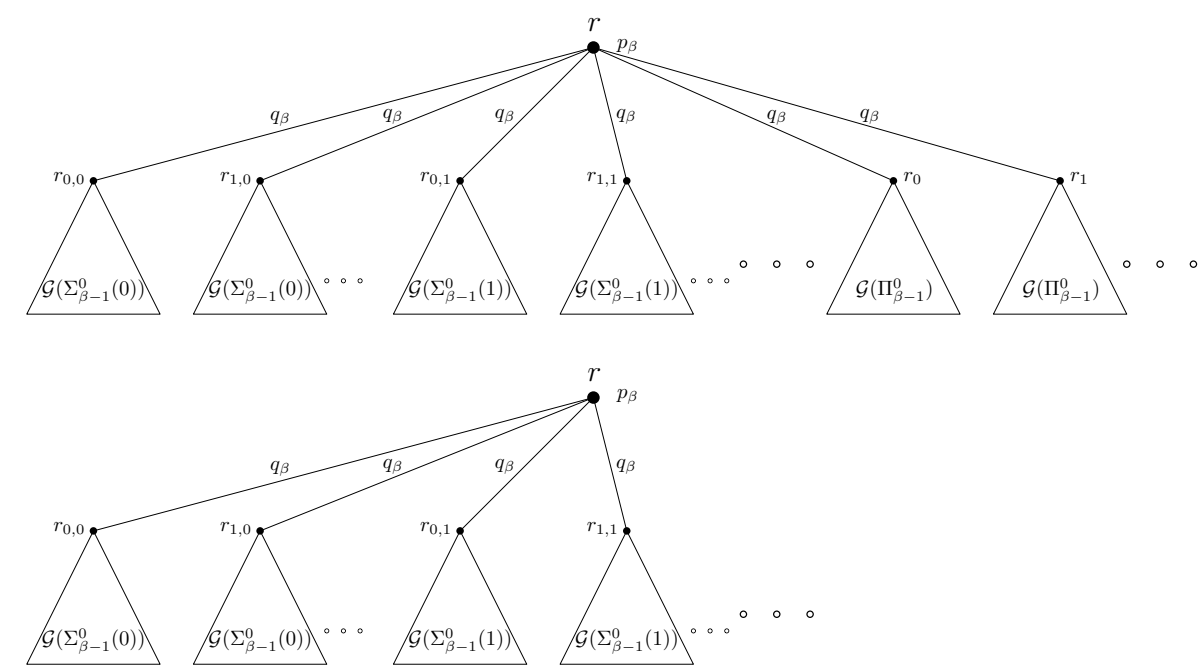

Figure 1. $\mathcal{G}\left(\Sigma_{\beta}^{0}\right)$ (Top) and $\mathcal{G}\left(\Pi_{\beta}^{0}\right)$ (Bottom) if $\beta=2 \delta+2 \ell+1>1$
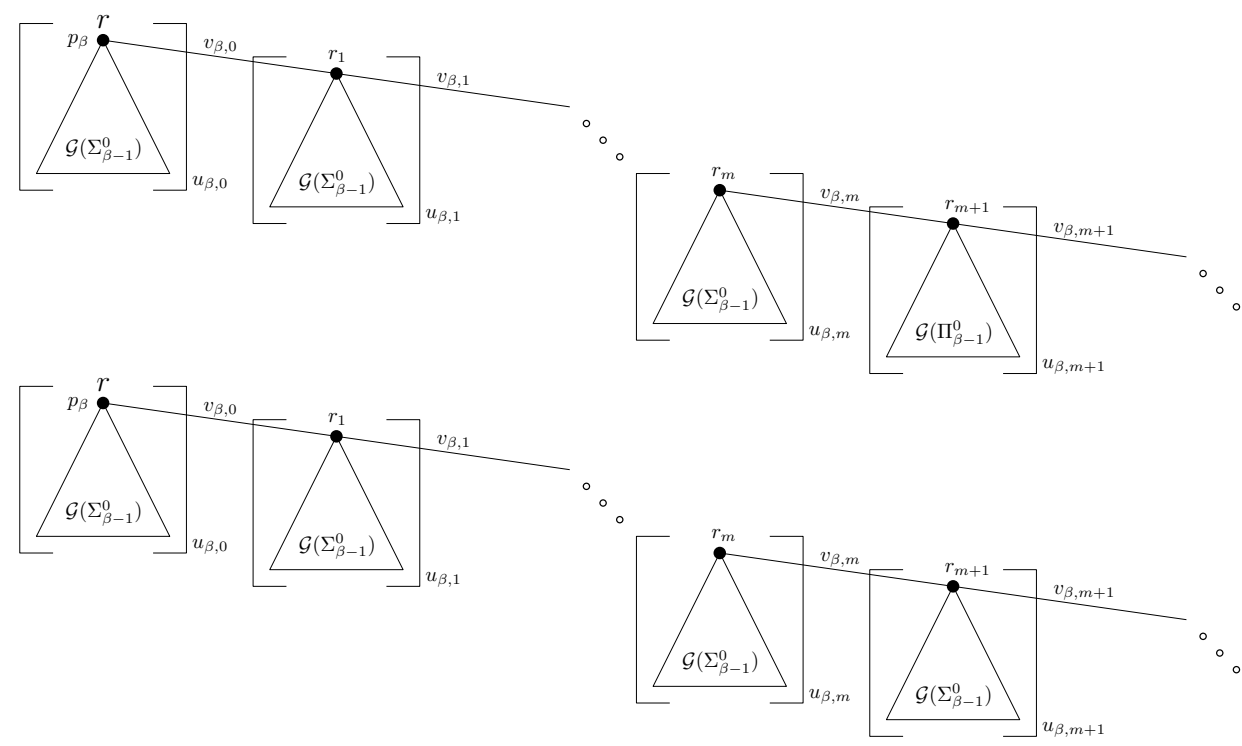

Figure 2. $\mathcal{G}\left(\Sigma_{\beta}^{0}(m)\right)$ (Top) and $\mathcal{G}\left(\Pi_{\beta}^{0}\right)$ (Bottom) if $\beta=\delta+2 \ell+2>2$

with root $r=1$ in $[\mathbb{Z}]_{p_{\beta}}$, where $r_{k}$ is the root of the $k$ th copy of $\mathcal{G}\left(\Pi_{\beta-1}^{0}\right)$ and $r_{k, m}$ is the root of $k$ th copy of $\mathcal{G}\left(\Sigma_{\beta-1}^{0}(m)\right)$.

For odd $\beta=\delta+2 \ell+1 \geq 3$, define $\mathcal{G}\left(\Pi_{\beta}^{0}\right)$ to be the group

$$
\left\langle[\mathbb{Z}]_{p_{\beta}} \oplus \bigoplus_{k \in \omega} \bigoplus_{m \in \omega} \mathcal{G}\left(\Sigma_{\beta-1}^{0}(m)\right) ; q_{\beta}^{-t}\left(r+r_{k, m}\right): k, m, t \in \omega\right\rangle,
$$

with root $r=1 \mathrm{in}[\mathbb{Z}]_{p_{\beta}}$, where $r_{k, m}$ is the root of $k$ th copy of $\mathcal{G}\left(\Sigma_{\beta-1}^{0}(m)\right)$.

These are illustrated in Figure 1. 

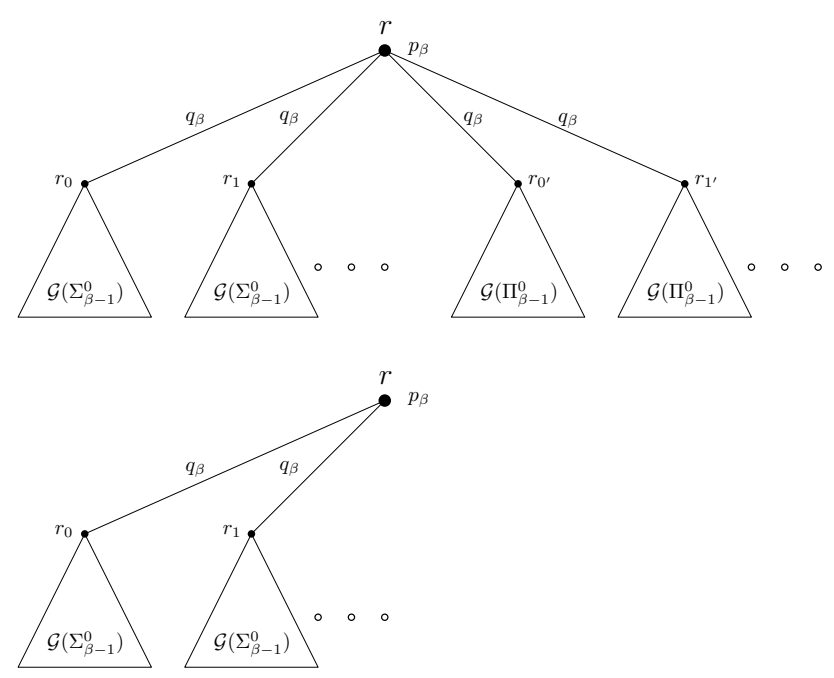

Figure 3. $\mathcal{H}\left(\Sigma_{\beta}^{0}\right)$ (Top) and $\mathcal{H}\left(\Pi_{\beta}^{0}\right)$ (Bottom) if $\beta=\delta+2 \ell+2>2$

- For even $\beta=\delta+2 \ell+2>2$, define $\mathcal{G}\left(\Sigma_{\beta}^{0}(m)\right)$ to be the group

$\left\langle\bigoplus_{0 \leq k \leq m}\left[\mathcal{G}\left(\Sigma_{\beta-1}^{0}\right)\right]_{u_{\beta, k}} \oplus \bigoplus_{k>m}\left[\mathcal{G}\left(\Pi_{\beta-1}^{0}\right)\right]_{u_{\beta, k}} ; p_{\beta}^{-t} r_{0}, v_{\beta, k}^{-t}\left(r_{k}+r_{k+1}\right): k, t \in \omega\right\rangle$,

with root $r=r_{0}$, where $r_{k}$ is the root of the $k$ th copy of $\mathcal{G}\left(\Sigma_{\beta-1}^{0}\right)$ or of $\mathcal{G}\left(\Pi_{\beta-1}^{0}\right)$ depending on whether $k \leq m$ or $k>m$.

For even $\beta=\delta+2 \ell+2>2$, define $\mathcal{G}\left(\Pi_{\beta}^{0}\right)$ to be the group

$$
\left\langle\bigoplus_{k \in \omega}\left[\mathcal{G}\left(\Sigma_{\beta-1}^{0}\right)\right]_{u_{\beta, k}} ; p_{\beta}^{-t} r_{0}, v_{\beta, k}^{-t}\left(r_{k}+r_{k+1}\right): k, t \in \omega\right\rangle,
$$

with root $r=r_{0}$, where $r_{k}$ is the root of the $k$ th copy of $\mathcal{G}\left(\Sigma_{\beta-1}^{0}\right)$.

These are illustrated in Figure 2.

- For limit $\beta=\delta>0$, define the group $\mathcal{G}\left(\Sigma_{\beta}^{0}(m)\right)$ to be

$$
\left\langle\bigoplus_{0 \leq k \leq m}\left[\mathcal{G}\left(\Sigma_{f_{\beta}(k)}^{0}\right)\right]_{u_{\beta, k}} \oplus \bigoplus_{k>m}\left[\mathcal{G}\left(\Pi_{f_{\beta}(k)}^{0}\right)\right]_{u_{\beta, k}} ; p_{\beta}^{-t} r_{0}, v_{\beta, k}^{-t}\left(r_{k}+r_{k+1}\right): k, t \in \omega\right\rangle,
$$

with root $r=r_{0}$, where $r_{k}$ is the root of the $k$ th copy of $\mathcal{G}\left(\Sigma_{f_{\beta}(k)}^{0}\right)$ or of $\mathcal{G}\left(\Pi_{f_{\beta}(k)}^{0}\right)$ depending on whether $k \leq m$ or $k>m$.

Define $\mathcal{G}\left(\Pi_{\beta}^{0}\right)$ to be the group

$$
\left\langle\bigoplus_{k \in \omega}\left[\mathcal{G}\left(\Sigma_{f_{\beta}(k)}^{0}\right)\right]_{u_{\beta, k}} ; p_{\beta}^{-t} r_{0}, v_{\beta, k}^{-t}\left(r_{k}+r_{k+1}\right): k, t \in \omega\right\rangle,
$$

with root $r=r_{0}$, where $r_{k}$ is the root of the $k$ th copy of $\mathcal{G}\left(\Sigma_{f_{\beta}(k)}^{0}\right) .^{3}$

\footnotetext{
${ }^{3}$ We emphasize that the definition of $\mathcal{G}\left(\Sigma_{\beta}^{0}(m)\right)$ and $\mathcal{G}\left(\Pi_{\beta}^{0}\right)$ for $\beta=\delta+2 \ell+2$ is identical to the case of $\beta=\delta$ as by definition $f_{\delta+2 \ell+2}(k)=\delta+2 \ell+1$ for all $k$. We separate them, here and in some later proofs, in hopes of not obfuscating the intuition.
} 
This completes the formal descriptions of these groups.

For odd $\beta \geq 3$, the group $\mathcal{G}\left(\Sigma_{\beta}^{0}\right)$ has the form

$$
\left\langle[\mathbb{Z}]_{p_{\beta}} \oplus \bigoplus_{k \in \omega} \bigoplus_{m \in \omega} \mathcal{G}\left(\Sigma_{\beta-1}^{0}(m)\right) \oplus \bigoplus_{k \in \omega} \mathcal{G}\left(\Pi_{\beta-1}^{0}\right) ; q_{\beta}^{-t}\left(r+r_{k}\right), q_{\beta}^{-t}\left(r+r_{k, m}\right): k, m, t \in \omega\right\rangle .
$$

We refer to the subgroups (indexed by $k$ and $m$ ) of the form $\mathcal{G}\left(\Sigma_{\beta-1}^{0}(m)\right.$ ) with roots $r_{k, m}$ as the $\mathcal{G}\left(\Sigma_{\beta-1}^{0}(m)\right)$ components of $\mathcal{G}\left(\Sigma_{\beta}^{0}\right)$. Similarly, we refer to the $\mathcal{G}\left(\Pi_{\beta-1}^{0}\right)$ subgroups (indexed by $k$ ) with root $r_{k}$ as the $\mathcal{G}\left(\Pi_{\beta-1}^{0}\right)$ components of $\mathcal{G}\left(\Sigma_{\beta}^{0}\right)$. We use similar language in the case of even $\beta$ and limit $\beta$, as well as for other groups defined inductively within the paper.

We emphasize the components of a group do not detach as direct summands. For clarity, we always refer to direct components (when the directness is an issue) without omitting the word "direct".

When we speak of components, we mean the components which are used in the inductive definition of these groups (or their prime closures), and we do not care if there are alternate ways to present the group. More formally, every such group will be considered as an image of one canonical copy given by the definition, and a subgroup is a component if and only if it is an image of a component which was used in the definition of this canonical copy. The isomorphism is chosen once and forever.

The important relationship between $\mathcal{G}\left(\Sigma_{\beta}^{0}\right)$ and $\mathcal{G}\left(\Pi_{\beta}^{0}\right)$ for odd $\beta$ and $\mathcal{G}\left(\Sigma_{\beta}^{0}(m)\right)$ and $\mathcal{G}\left(\Pi_{\beta}^{0}\right)$ for even $\beta$ is whether each embeds within the other. For small $\beta$, one can see that the groups defined above satisfy the following embeddability relations: if $\beta>1$ is odd, then $\mathcal{G}\left(\Sigma_{\beta}^{0}\right) \not \mathcal{G}\left(\Pi_{\beta}^{0}\right)$ and $\mathcal{G}\left(\Pi_{\beta}^{0}\right) \leqq \mathcal{G}\left(\Sigma_{\beta}^{0}\right)$; if $\beta>0$ is even, then $\mathcal{G}\left(\Pi_{\beta}^{0}\right) \geq \mathcal{G}\left(\Sigma_{\beta}^{0}(m)\right)$ and $\mathcal{G}\left(\Sigma_{\beta}^{0}(m)\right) \leqq \mathcal{G}\left(\Pi_{\beta}^{0}\right)$ for all $m \in \omega$. For larger ordinals $\beta$, the formal proof of these properties is less straightforward. Moreover, stronger properties of such groups are needed to run a successful induction. We avoid these formal difficulties by not using these embedability relations in later proofs, stating them only in order to aid intuition. Though they will not be formally used, the reader may find it useful to keep in mind which groups are "bigger".

The embeddability relations discussed reflect the utility of the coding. Informally, we will ask

\section{Is there a large subgroup attached to $x$ ?}

about an element $x$ that is infinitely divisible by an appropriate prime. The answer will allow us to extract whether the $\Sigma_{\beta}^{0}$ outcome or the $\Pi_{\beta}^{0}$ outcome was the case.

We (informally) justify not using a simpler recursive scheme to define the groups $\mathcal{G}\left(\Sigma_{\beta}^{0}\right)$ and $\mathcal{G}\left(\Pi_{\beta}^{0}\right)$ in the following remark.

Remark 3.2. It would of course be simpler if Definition 3.1 used only the odd recursion schema (for all successor ordinals). Unfortunately, the embeddability relations would not be satisfied in this case, e.g., when $\beta=4$ it would be the case that $\mathcal{G}\left(\Sigma_{\beta}^{0}\right) \leqq \mathcal{G}\left(\Pi_{\beta}^{0}\right)$ and $\mathcal{G}\left(\Pi_{\beta}^{0}\right) \leqq \mathcal{G}\left(\Sigma_{\beta}^{0}\right)$. The reason is $\mathcal{G}\left(\Sigma_{4}^{0}\right)$ would contain infinitely many copies of $\mathcal{G}\left(\Pi_{3}^{0}\right)$ and infinitely many copies of $\mathcal{G}\left(\Sigma_{3}^{0}\right)$ whereas $\mathcal{G}\left(\Pi_{4}^{0}\right)$ only would contain infinitely many copies of $\mathcal{G}\left(\Sigma_{3}^{0}\right)$. As $\mathcal{G}\left(\Pi_{3}^{0}\right) \leqq \mathcal{G}\left(\Sigma_{3}^{0}\right)$, it would follow that $\mathcal{G}\left(\Sigma_{4}^{0}\right) \leq \mathcal{G}\left(\Pi_{4}^{0}\right)$. Hence asking if there is a large subgroup would not distinguish between the $\Sigma_{\beta}^{0}$ and the $\Pi_{\beta}^{0}$ outcomes. 
For even successor ordinals $\beta \geq 4$, we will need additional auxiliary groups $\mathcal{H}\left(\Sigma_{\beta}^{0}\right)$ and $\mathcal{H}\left(\Pi_{\beta}^{0}\right)$.

Definition 3.3. For each even computable ordinal $\beta=\delta+2 \ell+2 \geq 4$, define rooted torsion-free abelian groups $\mathcal{H}\left(\Sigma_{\beta}^{0}\right)$ and $\mathcal{H}\left(\Pi_{\beta}^{0}\right)$ as follows.

Define $\mathcal{H}\left(\Sigma_{\beta}^{0}\right)$ to be the group

$$
\left\langle[\mathbb{Z}]_{p_{\beta}} \oplus \bigoplus_{k \in \omega} \mathcal{G}\left(\Sigma_{\beta-1}^{0}\right) \oplus \bigoplus_{k^{\prime} \in \omega} \mathcal{G}\left(\Pi_{\beta-1}^{0}\right) ; q_{\beta}^{-t}\left(r+r_{k}\right), q_{\beta}^{-t}\left(r+r_{k^{\prime}}\right): k, k^{\prime}, t \in \omega\right\rangle,
$$

with root $r=1$ in $[\mathbb{Z}]_{p_{\beta}}$, where $r_{k}$ is the root of the $k$ th copy of $\mathcal{G}\left(\Sigma_{\beta-1}^{0}\right)$ and $r_{k^{\prime}}$ is the root of $k^{\prime}$ th copy of $\mathcal{G}\left(\Pi_{\beta-1}^{0}\right)$.

Define $\mathcal{H}\left(\Pi_{\beta}^{0}\right)$ to be the group

$$
\left\langle[\mathbb{Z}]_{p_{\beta}} \oplus \bigoplus_{k \in \omega} \mathcal{G}\left(\Sigma_{\beta-1}^{0}\right) ; q_{\beta}^{-t}\left(r+r_{k}\right): k, t \in \omega\right\rangle
$$

with root $r=1$ in $[\mathbb{Z}]_{p_{\beta}}$, where $r_{k}$ is the root of the $k$ th copy of $\mathcal{G}\left(\Sigma_{\beta-1}^{0}\right)$.

These are illustrated in Figure 3.

It is now possible to define $\mathcal{G}_{S}$ for $S \subseteq \omega$.

Definition 3.4. For each successor ordinal $\alpha \geq 3$ and set $S \subseteq \omega$, define a torsionfree abelian group $\mathcal{G}_{S}=\mathcal{G}_{S}^{\alpha}$ as follows.

- If $\alpha=\delta+2 \ell+1 \geq 3$, define $\mathcal{G}_{S}$ to be the group

$$
\mathcal{G}_{S}:=\bigoplus_{n \in S}\left[\mathcal{G}\left(\Sigma_{\alpha}^{0}\right)\right]_{d_{n}} \oplus \bigoplus_{n \notin S}\left[\mathcal{G}\left(\Pi_{\alpha}^{0}\right)\right]_{d_{n}} .
$$

- If $\alpha=\delta+2 \ell+2$, define $\mathcal{G}_{S}$ to be the group

$$
\mathcal{G}_{S}:=\bigoplus_{n \in S}\left[\mathcal{H}\left(\Sigma_{\alpha}^{0}\right)\right]_{d_{n}} \oplus \bigoplus_{n \notin S}\left[\mathcal{H}\left(\Pi_{\alpha}^{0}\right)\right]_{d_{n}} .
$$

The following crucial observation will be exploited in later sections when we wish to express elements as sums of roots of subcomponents.

Remark 3.5. If $\mathcal{G}$ is any group within this section, or any direct product of prime closures of such groups, then we let $R_{\mathcal{G}}$ denote the set of (distinct) roots of the recursively nested components of $\mathcal{G}$. (Some elements may serve as the root of more than one component at different ordinal levels. For example, if $\beta$ is odd, then the root of a $\mathcal{G}\left(\Sigma_{\beta-1}^{0}(m)\right)$ component of $\mathcal{G}\left(\Pi_{\beta}^{0}\right)$ is also the root of a $\left[\mathcal{G}\left(\Sigma_{\beta-2}^{0}\right)\right]_{u_{\beta-1,0}}$ component. However, this root appears only once in $R_{\mathcal{G}}$.) The important point is that the set $R_{\mathcal{G}}$ is a basis for both $\mathcal{G}$ and $D(\mathcal{G})$.

\section{Proof of Theorem 1.5}

Having defined $\mathcal{G}_{S}$ for each successor ordinal $\alpha \geq 3$, it of course remains to verify the desired properties. We state these explicitly.

Lemma 4.1. For each successor ordinal $\alpha \geq 3$, there is an effective enumeration $\left\{\Upsilon_{n}\right\}_{n \in \omega}$ of computable $\Sigma_{\alpha}^{c}$ sentences such that $\mathcal{G}_{S} \models \Upsilon_{n}$ if and only if $n \in S$.

Lemma 4.2. For each successor ordinal $\alpha \geq 3$, if $S \in \Sigma_{\alpha}^{0}(X)$, then $\mathcal{G}_{S}$ has an $X$-computable copy. 
Assuming Lemma 4.1 and Lemma 4.2, we prove Theorem 1.5. Lemma 4.1 is demonstrated in Section 4.1 and Lemma 4.2 is demonstrated in Section 4.2. We note the proof of Theorem 1.5 from Lemmas 4.1 and 4.2 is identical to the context of linear orders (see [1]).

Proof of Theorem 1.5. Fix a computable ordinal $\alpha$, a degree $\mathbf{a}>\mathbf{0}^{(\alpha)}$, and a set $A \in$ a. By Theorem 1.4, we may assume that $\alpha \geq 3$.

If $\alpha$ is a successor ordinal $\beta+1$, we argue the torsion-free abelian group $\mathcal{G}:=$ $\mathcal{G}_{A \oplus \bar{A}}$ has proper $\alpha^{t h}$ jump degree a. By Lemma 4.1 and Lemma 4.2, we have

$$
\begin{aligned}
\operatorname{DegSpec}(\mathcal{G}) & =\left\{X: A \oplus \bar{A} \in \Sigma_{\alpha}^{0}(X)\right\} \\
& =\left\{X: A \in \Delta_{\alpha}^{0}(X)\right\}
\end{aligned}
$$

(for $\alpha$ finite, these are $\Sigma_{\alpha+1}^{0}(X)$ and $\Delta_{\alpha+1}^{0}(X)$ ). It follows $\left\{X^{(\alpha)}: X \in \operatorname{DegSpec}(\mathcal{G})\right\}$ contains precisely those sets that compute $A$. Thus $\mathcal{G}$ has $\alpha^{\text {th }}$ jump degree a. On the other hand, if $\beta<\alpha$, the set $\left\{X^{(\beta)}: X \in \operatorname{DegSpec}(\mathcal{G})\right\}$ has no element of least degree (see Lemma 1.3 of [1]). Thus $\mathcal{G}$ does not have $\beta^{\text {th }}$ jump degree for any $\beta<\alpha$.

If $\alpha$ is a limit ordinal, fix an $\alpha$-generic set $B$ such that $B^{(\alpha)} \equiv_{T} B \oplus \emptyset^{(\alpha)} \equiv_{T} A$. Viewing $B$ as a subset of $\omega \times \omega$, we write $B_{n}:=\{k:(n, k) \in B\}$. We argue the torsion-free abelian group

$$
\mathcal{G}:=\bigoplus_{n \in \omega}\left[\mathcal{G}_{B_{n}}\right]_{e_{n}}
$$

has proper $\alpha^{t h}$ jump degree a, where $\mathcal{G}_{B_{n}}$ is the group associated with the set $B_{n}$ and the ordinal $f_{\alpha}(n)$. Making use of the uniformity in both Lemma 4.1 and Lemma 4.2 and that the prime $e_{n}$ distinguishes the subgroup $\mathcal{G}_{B_{n}}$ from $\mathcal{G}_{B_{n^{\prime}}}$ for $n^{\prime} \neq n$, we have

$$
\operatorname{DegSpec}(\mathcal{G})=\left\{X: B_{n} \in \Sigma_{f_{\alpha}(n)}^{0}(X) \text { uniformly in } n\right\} .
$$

It follows $\left\{X^{(\alpha)}: X \in \operatorname{DegSpec}(\mathcal{G})\right\}$ contains precisely those sets that compute $B^{(\alpha)}$. Thus $\mathcal{G}$ has $\alpha^{\text {th }}$ jump degree $\mathbf{a}$. On the other hand, the set $\left\{X^{(\beta)}: X \in \operatorname{DegSpec}(\mathcal{G})\right\}$ has no element of least degree for any $\beta<\alpha$ (see discussion after Lemma 3.1 of [1]). Thus $\mathcal{G}$ does not have $\beta^{\text {th }}$ jump degree for any $\beta<\alpha$.

4.1. Proof of Lemma 4.1. The definition of the $\Sigma_{\alpha}^{c}$ sentences $\left\{\Upsilon_{n}\right\}_{n \in \omega}$ is done recursively, mirroring the recursive nature of the definition of $\mathcal{G}_{S}$. Before we start constructing formulas $\Phi_{\beta}(x)$ and $\Psi_{\beta}(x)$ connected semantically to $\mathcal{G}\left(\Sigma_{\beta}^{0}\right), \mathcal{G}\left(\Sigma_{\beta}^{0}(m)\right)$, and $\mathcal{G}\left(\Pi_{\beta}^{0}\right)$, we demonstrate two divisibility lemmas that isolate aspects of the odd and even inductive steps. The proofs of these are similar to proofs of lemmas by Downey and Montalbán (see Lemma 2.3 and Lemma 2.4 of [7]). For the proof of Lemma 4.4(1), we make explicit whether we are viewing elements of $\mathcal{B}$ as belonging to $\mathcal{B}$ or the divisible closure. For later parts of Lemma 4.4 and Lemma 4.5, we do not make it explicit as which it should be is clear from context. Before stating these two divisibility lemmas, we note a number of simple number theoretic facts (without proof) that we will use repeatedly (without mention).

Lemma 4.3. The following facts hold in prime closures of $\mathbb{Z}$.

- For any primes $p_{0}$ and $p_{1},[\mathbb{Z}]_{p_{0}}+[\mathbb{Z}]_{p_{1}}=[\mathbb{Z}]_{p_{0}, p_{1}}$. (The sum $[\mathbb{Z}]_{p_{0}}+[\mathbb{Z}]_{p_{1}}$ denotes the set of all $q \in \mathbb{Q}$ such that $q=a+b$ for some $a \in[\mathbb{Z}]_{p_{0}}$ and $\left.b \in[\mathbb{Z}]_{p_{1}}.\right)$ 
- For all sets of primes $P_{0}$ and $P_{1},[\mathbb{Z}]_{P_{0}} \cap[\mathbb{Z}]_{P_{1}}=[\mathbb{Z}]_{P_{0} \cap P_{1}}$.

- If $P_{0}$ and $P_{1}$ are disjoint sets of primes, then $\left([\mathbb{Z}]_{P_{0}} \backslash \mathbb{Z}\right) \cap[\mathbb{Z}]_{P_{1}}=\emptyset$ and $0 \notin\left([\mathbb{Z}]_{P_{0}} \backslash \mathbb{Z}\right)+[\mathbb{Z}]_{P_{1}}$.

Lemma 4.4. Fix pairwise disjoint sets of prime numbers $F_{1}, F_{2}$ and $P$ and fix a prime number $\rho \notin F_{1} \cup F_{2} \cup P$. For each $i \in \omega$, fix a copy of $[\mathbb{Z}]_{F_{1}}$ and let $x_{i}$ denote the element 1 in this copy. For each $i, j \in \omega$, fix a copy of $[\mathbb{Z}]_{F_{2}}$ and let $y_{i, j}$ denote the element 1 in this copy. Let $\mathcal{B}$ be the group

$$
\mathcal{B}:=\left[\left\langle\bigoplus_{i \in \omega}[\mathbb{Z}]_{F_{1}} \oplus \bigoplus_{i, j \in \omega}[\mathbb{Z}]_{F_{2}} ; \frac{x_{i}+y_{i, j}}{\rho^{k}}: i, j, k \in \omega\right\rangle\right]_{P}
$$

Then $\mathcal{B}$ has the following properties:

(1) For any $z \in B$ and $\sigma_{1} \in F_{1}$, we have $\sigma_{1}^{\infty} \mid z$ if and only if $z=\sum_{i} m_{i} x_{i}$ with $m_{i} \in[\mathbb{Z}]_{F_{1}, P}$.

(2) For any $y \in B$ and $\sigma_{2} \in F_{2}$, we have $\sigma_{2}^{\infty} \mid y$ if and only if $y=\sum_{i, j} m_{i, j} y_{i, j}$ with $m_{i, j} \in[\mathbb{Z}]_{\rho, F_{2}, P}$.

(3) Fixing $\ell$, if $\rho^{\infty} \mid \sum_{j} m_{\ell, j} y_{\ell, j}$, then $\sum_{j} m_{\ell, j}=0$.

(4) If $z$ can be expressed as $z=\sum m_{i} x_{i}$ with $m_{i} \in[\mathbb{Z}]_{P}$, then for each $\sigma_{1} \in F_{1}$ and $\sigma_{2} \in F_{2}, z$ satisfies the formula

$$
\sigma_{1}^{\infty} \mid z \wedge(\exists y \in B)\left[\rho^{\infty}\left|(z+y) \wedge \sigma_{2}^{\infty}\right| y\right] .
$$

(5) If $z \in B$ satisfies (†) with witness $y \in B$, then $z=\sum_{i} m_{i} x_{i}$ with $m_{i} \in[\mathbb{Z}]_{P}$ and $y=\sum_{i, j} m_{i, j} y_{i, j}$ with $m_{i, j} \in[\mathbb{Z}]_{\rho, F_{2}, P}$ for all $i, j$ and $m_{i}=\sum_{j} m_{i, j}$ for all $i$ (allowing the possibility of $m_{i}=0$ ).

Indeed, in any torsion-free abelian group of which $\mathcal{B}$ is a pure subgroup, these facts remain true.

Proof. For (1), the backward direction is immediate. For the forward direction, we express $z$ (in the divisible closure) as $z=\sum_{i} m_{i} x_{i}+\sum_{i, j} m_{i, j} y_{i, j}$ with $m_{i}, m_{i, j} \in \mathbb{Q}$ (allowing the possibility of a coefficient being zero). If $\sigma_{1}^{\infty} \mid z$, as the summation is finite, there is a $[\mathbb{Z}]_{\sigma_{1}}$-multiple $\hat{z}$ of $z$ in $\mathcal{B}$ with

$$
\hat{z}=\sum_{i} \frac{\hat{m}_{i}}{\sigma_{1}^{n_{i}}} x_{i}+\sum_{i, j} \frac{\hat{m}_{i, j}}{\sigma_{1}^{n_{i, j}}} y_{i, j}
$$

(with the right hand side expressed in the divisible closure) where $\hat{m}_{i}, \hat{m}_{i, j} \in \mathbb{Z}$, $\hat{m}_{i} \neq 0$ implies $\sigma_{1} \backslash \hat{m}_{i}, \hat{m}_{i, j} \neq 0$ implies $\sigma_{1} \backslash \hat{m}_{i, j}$, and $n_{i}, n_{i, j}>0$. Since the coefficient of $y_{i, j}$ in any element of $\mathcal{B}$ (viewed in the divisible closure) is an element of $[\mathbb{Z}]_{\rho, F_{2}, P^{4}}$ and $\left([\mathbb{Z}]_{\sigma_{1}} \backslash \mathbb{Z}\right) \cap\left([\mathbb{Z}]_{\rho, F_{2}, P}\right)=\emptyset$, it must be that $\hat{m}_{i, j}=0$ for all $i, j$, and so $m_{i, j}=0$ for all $i, j$. The reason that the coefficient of $y_{i, j}$ in any element of $\mathcal{B}$ (viewed in the divisible closure) is an element of $[\mathbb{Z}]_{\rho, F_{2}, P}$ is an immediate consequence of the fact that every element of $\mathcal{B}$ is a formal sum $\sum_{i} a_{i} x_{i}+\sum_{i, j} b_{i, j}\left(x_{i}+y_{i, j}\right)+\sum_{i, j} c_{i, j} y_{i, j}$ with $a_{i} \in[\mathbb{Z}]_{F_{1}, P}, b_{i, j} \in[\mathbb{Z}]_{\rho, P}$, and $c_{i, j} \in[\mathbb{Z}]_{F_{2}, P}$. Thus, in the divisible closure, the coefficient of any fixed $y_{i, j}$ is an element of $[\mathbb{Z}]_{\rho, P}+[\mathbb{Z}]_{F_{2}, P}=[\mathbb{Z}]_{\rho, F_{2}, P}$.

Thus if $\sigma_{1}^{\infty} \mid z$, then $z=\sum_{i} m_{i} x_{i}$ (in the divisible closure) with $m_{i} \in \mathbb{Q}$. From the structure of elements of $\mathcal{B}$, we have $m_{i} \in[\mathbb{Z}]_{\rho, F_{1}, P}$. Fix $i$. If $m_{i} \notin[\mathbb{Z}]_{F_{1}, P}$, then there would be a non- $[\mathbb{Z}]_{P}$-multiple of $x_{i}+y_{i, j}$ in $z$ for some $j$, in particular a

\footnotetext{
${ }^{4}$ Though we justify this here, we omit such arguments in the rest of the paper as all are similar to the argument here.
} 
$[\mathbb{Z}]_{\rho, P} \backslash[\mathbb{Z}]_{P}$-multiple. Then the coefficient of this $y_{i, j}$ in $z$ (in the divisible closure) would be in $[\mathbb{Z}]_{\rho, P} \backslash[\mathbb{Z}]_{P}+[\mathbb{Z}]_{F_{2}, P}$. However $0 \notin[\mathbb{Z}]_{\rho, P} \backslash[\mathbb{Z}]_{P}+[\mathbb{Z}]_{F_{2}, P}$, yielding a contradiction to the form $z=\sum_{i} m_{i} x_{i}$. Thus $m_{i} \in[\mathbb{Z}]_{F_{1}, P}$ for all $i$ completing proof of (1).

For (2), the argument is similar and we leave the minor change in details to the reader.

For (3), as $\rho^{\infty} \mid \sum_{j} m_{\ell, j} y_{\ell, j}$, there is a $[\mathbb{Z}]_{\rho}$-multiple $\hat{z}$ of $\sum_{j} m_{\ell, j} y_{\ell, j}$ in $\mathcal{B}$ with

$$
\hat{z}=\sum_{j} \frac{\hat{m}_{\ell, j}}{\rho_{2}^{n_{\ell, j}}} y_{\ell, j}
$$

where $\hat{m}_{\ell, j} \in \mathbb{Z}, \hat{m}_{\ell, j} \neq 0$ implies $\rho \mid \hat{m}_{\ell, j}$, and $n_{\ell, j}>0$. Indeed, we may assume $\sum_{j} \frac{\hat{m}_{\ell, j}}{\rho_{2} \ell, j} \notin[\mathbb{Z}]_{P}$ if $\sum_{j} m_{\ell, j} \neq 0$. From the structure of elements of $\mathcal{B}$, we have

$$
\hat{z}=\mu_{\ell} x_{\ell}+\sum_{j} \mu_{\ell, j}\left(x_{\ell}+y_{\ell, j}\right)+\sum_{j} \mu_{\ell, j}^{\prime} y_{\ell, j}
$$

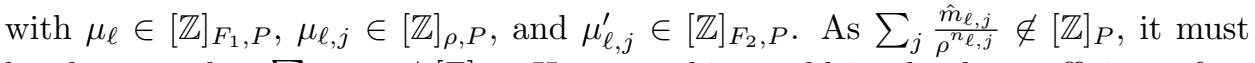
be the case that $\sum_{j} \mu_{\ell, j} \notin[\mathbb{Z}]_{P}$. However this would imply the coefficient of $x_{\ell}$ is nonzero as $0 \notin[\mathbb{Z}]_{F_{1}, P}+[\mathbb{Z}]_{\rho, P} \backslash[\mathbb{Z}]_{P}$. This would contradict the form of $\hat{z}$, showing (3).

For (4), we note if $z=\sum_{i} m_{i} x_{i}$ with $m_{i} \in[\mathbb{Z}]_{P}$, then $y=\sum_{i} m_{i} y_{i, 0}$ is in $\mathcal{B}$. Moreover, by Parts (1) and (2), this $y$ witnesses $z$ satisfying ( $\dagger$ ), showing (4).

For (5), fix $z$ and $y$ with $\sigma_{1}^{\infty}\left|z, \rho^{\infty}\right| z+y$, and $\sigma_{2}^{\infty} \mid y$. By Part (1), $z=\sum_{i} m_{i} x_{i}$ with $m_{i} \in[\mathbb{Z}]_{F_{1}, P}$ and by Part (2), $y=\sum_{i, j} m_{i, j} y_{i, j}$ with $m_{i, j} \in[\mathbb{Z}]_{\rho, F_{2}, P}$. As $\rho^{\infty} \mid z+y$, there is a $[\mathbb{Z}]_{\rho}$-multiple $\hat{z}+\hat{y}$ of $z+y$ in $\mathcal{B}$ with

$$
\hat{z}+\hat{y}=\sum_{i} \frac{\hat{m}_{i}}{\rho^{n_{i}}} x_{i}+\sum_{i, j} \frac{\hat{m}_{i, j}}{\rho^{n_{i, j}}} y_{i, j}
$$

where $\hat{m}_{i}, \hat{m}_{i, j} \in \mathbb{Z}, \hat{m}_{i} \neq 0$ implies $\rho \nmid \hat{m}_{i}, \hat{m}_{i, j} \neq 0$ implies $\rho \nmid \hat{m}_{i, j}$, and $n_{i}, n_{i, j}>0$. From the proof of Part (4), we have

$$
\hat{w}:=\sum_{i} \frac{\hat{m}_{i}}{\rho^{n_{i}}} x_{i}+\sum_{i} \frac{\hat{m}_{i}}{\rho^{n_{i}}} y_{i, 0}
$$

is in $\mathcal{B}$ (by virtue of it being a sum of $[\mathbb{Z}]_{\rho}$-multiples of $x_{i}+y_{i, 0}$ ) and infinitely divisible by $\rho$. Thus the element

$$
\hat{z}+\hat{y}-\hat{w}=\sum_{i, j} \frac{\hat{m}_{i, j}}{\rho^{n_{i, j}}} y_{i, j}-\sum_{i} \frac{\hat{m}_{i}}{\rho^{n_{i}}} y_{i, 0}
$$

is in $\mathcal{B}$ and is infinitely divisible by $\rho$. By Part (3), this implies $\frac{\hat{m}_{i}}{\rho^{n_{i}}}=\sum_{j} \frac{\hat{m}_{i, j}}{\rho^{n_{i, j}}}$ for all $i$. This is equivalent to $m_{i}=\sum_{j} m_{i, j}$ for all $i$.

As $m_{i, j} \in[\mathbb{Z}]_{\rho, F_{2}, P}$ for all $i, j$, fixing $i$, the sum $\sum_{j} m_{i, j}$ is in $[\mathbb{Z}]_{\rho, F_{2}, P}$. As $[\mathbb{Z}]_{F_{1}, P} \cap[\mathbb{Z}]_{\rho, F_{2}, P}=[\mathbb{Z}]_{P}$, it follows $m_{i} \in[\mathbb{Z}]_{P}$ for all $i$. This shows (5).

Lemma 4.5. Fix pairwise disjoint sets of primes $F_{i}$, for $i \in \omega$, and $P$, and fix a sequence of distinct primes $\rho_{n}$, for $n \in \omega$, such that $\rho_{n} \notin\left(\cup_{i \in \omega} F_{i}\right) \cup P$ for each $n$. Let $\mathcal{B}$ be the group

$$
\mathcal{B}:=\left[\left\langle\mathcal{F} ; \frac{x_{i, j}}{\sigma_{i}^{k}}, \frac{x_{i, j}+x_{i+1, j}}{\rho_{i}^{k}}: i, j, k \in \omega \text { and } \sigma_{i} \in F_{i}\right\rangle\right]_{P}
$$


where $\mathcal{F}$ is the free abelian group on the elements $x_{i, j}$ for $i, j \in \omega$. Then $\mathcal{B}$ has the following properties:

(1) Fixing $\ell$, a prime $\sigma_{\ell} \in F_{\ell}$ and $z \in B$, if $\sigma_{\ell}^{\infty} \mid z$, then $z=\sum_{j} m_{\ell, j} x_{\ell, j}$ with $m_{\ell, j} \in[\mathbb{Z}]_{F_{\ell}, P}$.

(2) Fixing $\ell$, if $z=\sum_{j} m_{\ell, j} x_{\ell, j}$ is nonzero, then $\rho_{i}^{\infty} \chi_{z}$ for any $i$.

(3) Fixing primes $\sigma_{i} \in F_{i}$ for $0 \leq i \leq k+1$, if $z_{0}, \ldots, z_{k+1} \in B$ satisfy

$$
\sigma_{i}^{\infty} \mid z_{i} \text { for all } i \leq k+1 \text { and } \rho_{i}^{\infty} \mid\left(z_{i}+z_{i+1}\right) \text { for all } i \leq k
$$

then there are constants $m_{j} \in[\mathbb{Z}]_{P}$ such that $z_{i}=\sum_{j} m_{j} x_{i, j}$ for all $0 \leq$ $i \leq k+1$.

Indeed, in any torsion-free abelian group of which $\mathcal{B}$ is a pure subgroup, these facts remain true.

Proof. For (1), we express $z$ as $z=\sum_{i, j} m_{i, j} x_{i, j}$ with $m_{i, j} \in \mathbb{Q}$. As $\sigma_{\ell}^{\infty} \mid z$ and the summation is finite, there is a $[\mathbb{Z}]_{\sigma_{\ell}}$-multiple $\hat{z}$ of $z$ in $\mathcal{B}$ with

$$
\hat{z}=\sum_{i, j} \frac{\hat{m}_{i, j}}{\sigma_{\ell}^{n_{i, j}}} x_{i, j}
$$

where $\hat{m}_{i, j} \in \mathbb{Z}, \hat{m}_{i, j} \neq 0$ implies $\sigma_{\ell} \nmid \hat{m}_{i, j}$, and $n_{i, j}>0$. Thus the coefficient of $x_{i, j}$ in $\hat{z}$ is an element of $[\mathbb{Z}]_{\sigma_{\ell}} \backslash \mathbb{Z}$. On the other hand, the coefficient of $x_{i, j}$ in any element of $\mathcal{B}$ is an element of $[\mathbb{Z}]_{F_{i}, P}+[\mathbb{Z}]_{\rho_{i}, P}+[\mathbb{Z}]_{\rho_{i-1}, P^{5}}$. As $\left([\mathbb{Z}]_{\sigma_{\ell}} \backslash \mathbb{Z}\right) \cap[\mathbb{Z}]_{F_{i}, \rho_{i}, \rho_{i-1}, P}=\emptyset$ if $i \neq \ell$, it follows that $z$ can be expressed as $z=\sum_{j} m_{\ell, j} x_{\ell, j}$ with $m_{\ell, j} \in \mathbb{Q}$.

We show $m_{\ell, j} \in[\mathbb{Z}]_{F_{\ell}, P}$ for all $j$. Fixing $j$, if $m_{\ell, j}$ were not in $[\mathbb{Z}]_{F_{\ell}, P}$, there would necessarily be a non- $[\mathbb{Z}]_{P}$-multiple of either $x_{\ell, j}+x_{\ell+1, j}$ or $x_{\ell-1, j}+x_{\ell, j}$ in $\hat{z}$. This implies the coefficient of $x_{\ell+1, j}$ or $x_{\ell-1, j}$ is nonzero in $\hat{z}$ as $0 \notin[\mathbb{Z}]_{\rho_{\ell}, P} \backslash[\mathbb{Z}]_{P}+$ $[\mathbb{Z}]_{\rho_{\ell+1}, P}+[\mathbb{Z}]_{F_{\ell+1}, P}$ and $0 \notin[\mathbb{Z}]_{\rho_{\ell}, P} \backslash[\mathbb{Z}]_{P}+[\mathbb{Z}]_{\rho_{\ell-1}, P}+[\mathbb{Z}]_{F_{\ell-1}, P}$. However this contradicts the form of $\hat{z}$, so it must be that $m_{\ell, j} \in[\mathbb{Z}]_{F_{\ell}, P}$, showing (1).

For (2), fix an $\ell$, an element $z=\sum_{j} m_{\ell, j} x_{\ell, j}$, and an integer $i$ towards a contradiction. As we are assuming $\rho_{i}^{\infty} \mid z$ for a contradiction, there is a $[\mathbb{Z}]_{\rho_{i}}$-multiple $\hat{z}$ of $z$ in $\mathcal{B}$ with

$$
\hat{z}=\sum_{j} \frac{\hat{m}_{\ell, j}}{\rho_{i}^{n_{\ell, j}}} x_{\ell, j}
$$

where $\hat{m}_{\ell, j} \in \mathbb{Z}, \hat{m}_{\ell, j} \neq 0$ implies $\rho_{i} \nmid \hat{m}_{\ell, j}$, and $n_{\ell, j}>0$. Fix $j$ and note that the coefficient of $x_{\ell, j}$ in $\hat{z}$ is an element of $[\mathbb{Z}]_{\rho_{i}} \backslash \mathbb{Z}$. On the other hand, the coefficient of $x_{\ell, j}$ in any element of $\mathcal{B}$ is an element of $[\mathbb{Z}]_{F_{\ell}, P}+[\mathbb{Z}]_{\rho_{\ell}, P}+[\mathbb{Z}]_{\rho_{\ell-1}, P}$. As $\left([\mathbb{Z}]_{\rho_{i}} \backslash \mathbb{Z}\right) \cap$ $\left([\mathbb{Z}]_{F_{\ell}, P}+[\mathbb{Z}]_{\rho_{\ell}, P}+[\mathbb{Z}]_{\rho_{\ell-1}, P}\right)=\emptyset$ if $\ell \notin\{i, i+1\}$, we must have $\ell \in\{i, i+1\}$. We show that either yields a contradiction.

If $\ell=i$, as $\left([\mathbb{Z}]_{\rho_{\ell}} \backslash \mathbb{Z}\right) \cap\left([\mathbb{Z}]_{F_{\ell}, P}+[\mathbb{Z}]_{\rho_{\ell-1}, P}\right)=\emptyset$, the term $x_{\ell, j}+x_{\ell+1, j}$ must have a nonzero coefficient in the expression of $\hat{z}$ as an element of $\mathcal{B}$. Indeed, this coefficient must be an element of $[\mathbb{Z}]_{\rho_{\ell}} \backslash \mathbb{Z}$ as the coefficient of $x_{\ell, j}$ in $\hat{z}$ is in $[\mathbb{Z}]_{\rho_{\ell}} \backslash \mathbb{Z}$. This implies the coefficient of $x_{\ell+1, j}$ in $\hat{z}$ is nonzero as $0 \notin\left([\mathbb{Z}]_{\rho_{\ell}} \backslash \mathbb{Z}\right)+[\mathbb{Z}]_{\rho_{\ell+1}, P}+[\mathbb{Z}]_{F_{\ell+1}, P}$, contradicting the form of $z$. If $\ell=i+1$, identical reasoning suffices to contradict the form of $z$. We have thus shown (2).

For (3), we induct on $k$. For $k=0$, by Part (1), we have $z_{i}=\sum_{j} m_{i, j} x_{i, j}$ with $m_{i, j} \in[\mathbb{Z}]_{F_{i}, P}$ for $i \in\{0,1\}$. As $\rho_{0}^{\infty} \mid z_{0}+z_{1}$, there is a $[\mathbb{Z}]_{\rho_{0}}$-multiple $\hat{z}$ of

${ }^{5}$ We ignore the degenerate case of $i=0$ as it is actually simpler. 
$z:=z_{0}+z_{1}$ where

$$
\hat{z}=\sum_{j} \frac{\hat{m}_{0, j}}{\rho_{0}^{n_{0, j}}} x_{0, j}+\sum_{j} \frac{\hat{m}_{1, j}}{\rho_{0}^{n_{1, j}}} x_{1, j}
$$

with $\rho_{0} \backslash \hat{m}_{0, j}, \rho_{0} \backslash \hat{m}_{1, j}, n_{0, j}>0$, and $n_{1, j}>0$. We rewrite $\hat{z}$ as

$$
\hat{z}=\sum_{j} \frac{\hat{m}_{0, j}}{\rho_{0}^{n_{0, j}}}\left(x_{0, j}+x_{1, j}\right)+\sum_{j} \frac{\hat{m}_{1, j}-\rho_{0}^{n_{1, j}-n_{0, j}} \hat{m}_{0, j}}{\rho_{0}^{n_{1, j}}} x_{1, j} .
$$

As the first summation and $\hat{z}$ are both in $\mathcal{B}$ and infinitely divisible by $\rho_{0}$, so is the second summation. By Part (2), the second summation must be zero. Thus $\hat{m}_{1, j} / \rho_{0}^{n_{1, j}}=\hat{m}_{0, j} / \rho_{0}^{n_{0, j}}$ for all $j$, and so $m_{0, j}=m_{1, j}$ for all $j$, with this value an element of $[\mathbb{Z}]_{F_{0}, P} \cap[\mathbb{Z}]_{F_{1}, P}=[\mathbb{Z}]_{P}$, completing the base case.

Assuming Part (3) for $k$, we show it true for $k+1$. As in the base case, write $z_{i}=\sum_{j} m_{i, j} x_{i, j}$ with $m_{i, j} \in[\mathbb{Z}]_{F_{i}, P}$ for $i \leq k+2$ by Part (1). By the induction hypothesis, for each fixed $j$, the values of $m_{i, j}$ for $0 \leq i \leq k+1$ are equal. Let $m_{j}$ denote this common value. Since $m_{j}$ is in $[\mathbb{Z}]_{F_{0}, P} \cap \cdots \cap[\mathbb{Z}]_{F_{k}, P}$, it must be in $[\mathbb{Z}]_{P}$. As $z_{k+2}=\sum_{j} m_{k+2, j} x_{k+2, j}$ with $m_{k+2, j} \in[\mathbb{Z}]_{F_{k+2}, P}$, the same analysis as in the base case implies $m_{k+2, j}=m_{k+1, j}=m_{j}$.

We continue by introducing various formulas that capture structural aspects of the groups. These formulas describe how group elements interact in terms of infinite divisibility by certain primes. When defining these formulas and verifying their properties, we often restrict quantification from ranging over all group elements to ranging only over those elements which are infinitely divisible by certain primes.

To make this notion precise, we define the (computable infinitary) language of infinite divisibility. The signature of this language is the same as the signature of the language of groups except that for each prime $p$, we add a relation symbol for the relation $p^{\infty} \mid x$. That is, we treat $p^{\infty} \mid t$ for each prime $p$ and term $t$ as an atomic statement. We build up formulas in this language in the standard computable infinitary manner.

For any formula $\varphi$ in the infinite divisibility language and any prime $q$, we define the relativized formula $\varphi^{q}$ by induction as follows.

$$
\begin{gathered}
\text { For atomic formulas }\left(p^{\infty} \mid t\right)^{q}={ }_{\operatorname{def}} p^{\infty} \mid t \text { and }\left(t_{0}=t_{1}\right)^{q}={ }_{\operatorname{def}}\left(t_{0}=t_{1}\right) \\
\qquad \begin{array}{c}
\left.\left(\bigwedge_{i} \beta_{i}\right)^{q}={ }_{\operatorname{def}} \bigwedge_{i} \beta_{i}^{q} \text { (and similarly for } \bigvee, \neg \text { and } \rightarrow\right) \\
((\exists x) \beta(x))^{q}={ }_{\operatorname{def}}(\exists x)\left[q^{\infty} \mid x \wedge \beta^{q}(x)\right] \\
((\forall x) \beta(x))^{q}={ }_{\operatorname{def}}(\forall x)\left[q^{\infty} \mid x \rightarrow \beta^{q}(x)\right]
\end{array}
\end{gathered}
$$

Thus, a formula $\varphi^{q}$ restricts all quantification to be over elements which are infinitely divisible by the prime $q$. The following lemma is a formal statement of this property.

Lemma 4.6. Let $\mathcal{G}$ be a torsion-free abelian group, let $q$ be a prime and let $\mathcal{G}_{q}$ be the subgroup consisting of the elements infinitely divisible by $q$. If $\mathcal{G}_{q}$ is a pure subgroup, then for any formula $\varphi(\bar{x})$ in the language of infinite divisibility and any parameters $\bar{a}$ from $\mathcal{G}_{q}$

$$
\mathcal{G} \models \varphi^{q}(\bar{a}) \Leftrightarrow \mathcal{G}_{q} \vDash \varphi(\bar{a})
$$


In particular, if $\mathcal{G}$ is $\{q\}$-closed, then $\mathcal{G}_{q}=\mathcal{G}$ and hence

$$
\mathcal{G} \models \varphi^{q}(\bar{a}) \Leftrightarrow \mathcal{G} \models \varphi(\bar{a})
$$

Proof. Suppose $\mathcal{G}_{q}$ is a pure subgroup. We proceed by induction on $\varphi(\bar{x})$. If $\varphi(\bar{x})$ is atomic, then $\varphi^{q}(\bar{a})$ is the same as $\varphi(\bar{a})$. If $\varphi(\bar{a})$ has the form $t_{0}(\bar{a})=t_{1}(\bar{a})$, then (1) follows because $\mathcal{G}_{q}$ is a subgroup. If $\varphi(\bar{a})$ has the form $p^{\infty} \mid t(\bar{a})$, then (1) follows because $\mathcal{G}_{q}$ is pure. The inductive cases for $\Lambda, \bigvee, \rightarrow$ and $\neg$ follow immediately by definition, leaving only the quantifier cases. It suffices to consider the case for $\exists$.

Suppose $\varphi^{q}(\bar{a})$ has the form $((\exists x) \beta(x, \bar{a}))^{q}$ and $\mathcal{G} \models(\exists x)\left[q^{\infty} \mid x \wedge \beta^{q}(x, \bar{a})\right]$ with a fixed witness $x$. Since $x$ is infinitely divisible by $q$, we have $x \in \mathcal{G}_{q}$. By the inductive hypothesis $\mathcal{G}_{q} \models \beta(x, \bar{a})$ and hence $\mathcal{G}_{q} \models(\exists x) \beta(x, \bar{a})$ as required. Conversely, suppose $\mathcal{G}_{q} \models(\exists x) \beta(x, \bar{a})$ with fixed witness $x \in \mathcal{G}_{q}$. By the inductive hypothesis, $\mathcal{G} \models \beta^{q}(x, \bar{a})$, and since every element of $\mathcal{G}_{q}$ is infinitely divisible by $q$, we have $q^{\infty} \mid x$. Therefore, $\mathcal{G} \models(\exists x)\left[q^{\infty} \mid x \wedge \beta^{q}(x, \bar{a})\right]$ as required.

Because the language of infinite divisibility is infinitary, we can express the relation $p^{\infty} \mid x$ using the standard formula $\varphi_{p}(x)$ given by

$$
\varphi_{p}(x):=\bigwedge_{k \in \omega}(\exists y)\left[p^{k} y=x\right]
$$

In any group, the atomic relation $p^{\infty} \mid x$ and the formula $\varphi_{p}(x)$ are satisfied by the same elements. Thus, we can always translate formulas in the language of infinite divisibility into formulas in the (computable infinitary) language of group theory. ${ }^{6}$ Notice, however, that some caution is required because the relativized formulas $\left(p^{\infty} \mid x\right)^{q}$ and $\varphi_{p}^{q}(x)$ are not necessarily satisfied by the same elements. The formula $\left(p^{\infty} \mid x\right)^{q}$ is satisfied by any element which is infinitely divisible by $p$, while $\varphi_{p}^{q}(x)$ is only satisfied by those $x$ which are infinitely divisible by $p$ using witnesses that are infinitely divisibly by $q$.

When we measure the quantifier complexity of a formula in the language of infinite divisibility, we will always means its complexity as a formula in the language of group theory. Given the remarks in the previous paragraph, we need to be careful how we translate relativized formulas in the language of infinite divisibility into formulas in the language of group theory for the purposes of measuring complexity. Thus, when we say a formula $\varphi^{q}$ (in the language of infinite divisibility) is in $\Sigma_{\beta}^{c}$ or $\Pi_{\beta}^{c}$, we mean that the following formula $\psi$ (in the language of group theory) is in the complexity class. First, use the inductive definition of relativized quantifiers to write $\varphi^{q}$ in an unrelativized form in the language of infinite divisibility. Second, replace each occurrence of an atomic formula $p^{\infty} \mid t$ in this unrelativized formula by the corresponding formula $\varphi_{p}(t)$ to obtain a formula $\psi$ in the language of group theory. By performing the translation in this order, we ensure that we do not add additional divisibility conditions on the witnesses for $p^{\infty} \mid t$ and thus each atomic fact $p^{\infty} \mid t$ remains $\Pi_{2}^{c}$ even if it is under the scope of a relativizing prime.

We need one further convention before giving our formulas. Note that this convention does not change the quantifier complexity of any formula.

Convention 4.7. When we quantify over group elements using $(\exists z)$ or $(\forall z)$, the quantification is restricted to nonzero group elements. Hence $(\exists z)[\ldots]$ is an abbreviation for $(\exists z)[z \neq 0 \wedge \ldots]$ and $(\forall z)[\ldots]$ is an abbreviation for $(\forall z)[z=0 \vee \ldots]$.

\footnotetext{
${ }^{6}$ As all of our languages are computable infinitary languages, we drop explicit reference to this fact from now on.
} 
In a similar manner, we regard each of the formulas $A_{\beta}(x), \Phi_{\beta}(x)$ and $\Psi_{\beta}(x)$ as having an additional conjunct of the form $x \neq 0$. In most cases, we could show that such a conjunct is unnecessary, but it easier to put it in and ignore the issue of the identity element. (The point of this convention is merely to keep our formulas a reasonable size and to avoid repeatedly stating assumptions that elements are not the identity.)

The formulas $A_{\beta}(x)$ below capture when an element $x$ is a sum of roots of $\mathcal{G}\left(\Sigma_{\beta}^{0}(m)\right.$ ) components (for even $\beta$ ). The formulas $\Phi_{\beta}(x)$ and $\Psi_{\beta}(x)$ capture when an element $x$ is a sum of roots of $\mathcal{G}\left(\Sigma_{\beta}^{0}\right)$ components and a sum of roots of $\mathcal{G}\left(\Pi_{\beta}^{0}\right)$ components, respectively.

Definition 4.8. For each even ordinal $\beta$, we let $A_{\beta}(x)$ be the computable infinitary formula $A_{\beta}(x):=p_{\beta}^{\infty} \mid x \wedge(\exists w)\left[u_{\beta, 1}^{\infty}\left|w \wedge v_{\beta, 0}^{\infty}\right|(x+w)\right]$.

Definition 4.9. For each ordinal $\beta$ with $\beta \geq 2$, we define computable infinitary formulas $\Phi_{\beta}(x)$ (for odd $\beta$ ) and $\Psi_{\beta}(x)$ (for even $\beta$ ) by recursion as follows.

- If $\beta=2$, define $\Psi_{\beta}(x)$ to be the formula $\Psi_{2}(x):=p_{1}^{\infty} \mid x$.

- If $\beta=3$, define $\Phi_{\beta}(x)$ to be the formula

$$
\Phi_{3}(x):=p_{3}^{\infty} \mid x \wedge(\exists y)\left[q_{3}^{\infty} \mid(x+y) \wedge \Psi_{2}(y)\right] .
$$

- If $\beta=\delta+2 \ell>2$, define $\Psi_{\beta}(x)$ to be the formula

$\Psi_{\beta}(x):=\bigwedge_{m \in \omega}\left(\exists x_{0}, \ldots, x_{m}\right)\left[x_{0}=x \wedge \bigwedge_{k \leq m} u_{\beta, k}^{\infty}\left|x_{k} \wedge \bigwedge_{k<m} v_{\beta, k}^{\infty}\right|\left(x_{k}+x_{k+1}\right) \wedge \Phi_{f_{\beta}(m)}^{u_{\beta, m}}\left(x_{m}\right)\right]$.

(Note that when $\beta$ is a successor, the last conjunct is $\Phi_{\beta-1}^{u_{\beta, m}}\left(x_{m}\right)$.)

- If $\beta=\delta+2 \ell+1>3$, define $\Phi_{\beta}(x)$ to be the formula

$$
\Phi_{\beta}(x):=p_{\beta}^{\infty} \mid x \wedge(\exists y)\left[q_{\beta}^{\infty} \mid(x+y) \wedge A_{\beta-1}(y) \wedge \Psi_{\beta-1}(y)\right] .
$$

Lemma 4.10. The complexity of $A_{\beta}(x)$ is $\Sigma_{3}^{c}$ (independent of $\beta$ ). If $\beta=\delta+2 \ell \geq 2$, then $\Psi_{\beta} \in \Pi_{\beta}^{c}$. If $\beta=\delta+2 \ell+1 \geq 3$, then $\Phi_{\beta} \in \Sigma_{\beta}^{c}$. Furthermore, the relativization of these formulas to any prime does not change their complexity.

Proof. These statements follow immediately from $p^{\infty} \mid x$ being $\Pi_{2}^{c}$ and induction.

Lemma 4.11. Let $\rho_{0}, \rho_{1}$ and $\rho_{2}$ be distinct prime numbers and let $\psi(x)$ be the formula $\rho_{0}^{\infty} \mid x \wedge(\exists y)\left[\rho_{2}^{\infty}\left|y \wedge \rho_{3}^{\infty}\right|(x+y)\right]$. The following properties hold for any prime $q$.

(1) If $\mathcal{G} \models \psi^{q}(x)$ for a fixed $x \in \mathcal{G}$ with witness $y$ and $\mathcal{H}$ is a pure subgroup of $\mathcal{G}$ with $x, y \in \mathcal{H}$, then $\mathcal{H}=\psi^{q}(x)$ with witness $y$.

(2) If $\mathcal{H} \models \psi^{q}(x)$ for a fixed $x \in \mathcal{H}$ with witness $y$ and $\mathcal{H}$ is a subgroup of $\mathcal{G}$, then $\mathcal{G} \models \psi^{q}(x)$ with the same witness.

In particular, these properties hold for $A_{\beta}(x)$.

Proof. Part (1) follows because any infinite divisibility statement about elements $x, y \in \mathcal{G}$ is true in any pure subgroup containing $x$ and $y$. Part (2) follows because any elements which are infinitely divisible in a subgroup are also infinitely divisible in the larger group.

More generally, we have the following fact about our formulas. 
Lemma 4.12. Let $\varphi(x)$ be a formula of the form $A_{\beta}(x), \Phi_{\beta}(x)$ or $\Psi_{\beta}(x)$. If $\mathcal{H}=\varphi(x)$ for some fixed $x \in \mathcal{H}$ and if $\mathcal{H}$ is a subgroup of $\mathcal{G}$, then $\mathcal{G} \models \varphi(x)$.

Proof. In the recursive construction of the formulas $A_{\beta}(x), \Phi_{\beta}(x)$ and $\Psi_{\beta}(x)$, we only impose positive infinite divisibility conditions on elements and witnesses. If $\mathcal{H}$ is a subgroup of $\mathcal{G}$, then each of the recursively defined witnesses to $\varphi(x)$ is in $\mathcal{G}$ and the infinite divisibility conditions are met. Therefore, $\mathcal{G}$ satisfies $\varphi(x)$ using the same sequence of witnesses.

The next lemma gives the key properties needed to verify that our construction succeeds.

Lemma 4.13. Fix an odd ordinal $\beta \geq 3$ and a set of primes $P$ disjoint from $\left\{p_{\rho}\right\}_{\rho \leq \beta} \cup\left\{q_{\rho}\right\}_{\rho \leq \beta} \cup\left\{u_{\rho, m}\right\}_{\rho \leq \beta, m \in \omega} \cup\left\{v_{\rho, m}\right\}_{\rho \leq \beta, m \in \omega}$. Let $\mathcal{G}$ be the group $\left[\oplus_{i \in \omega} \mathcal{C}_{i}\right]_{P}$, where each $\mathcal{C}_{i}$ is either isomorphic to $\mathcal{G}\left(\Sigma_{\beta}^{0}\right)$ or $\mathcal{G}\left(\Pi_{\beta}^{0}\right)$.

(1) If $\beta=3$, then $\mathcal{G} \models \Psi_{2}(y)$ if and only if $y$ can be expressed as $y=\sum a_{i} y_{i}$ with each $y_{i}$ a root of a $\mathcal{G}\left(\Pi_{2}^{0}\right)$ component and $a_{i} \in[\mathbb{Z}]_{p_{1}, q_{3}, P}$.

(2) For $\beta=\delta+2 \ell+1>3$,

(a) if $\mathcal{G} \models A_{\beta-1}(z) \wedge \Psi_{\beta-1}(z)$, then $z=\sum a_{i} z_{i}$ with $z_{i}$ a root of $a \mathcal{G}\left(\Pi_{\beta-1}^{0}\right)$ component, $a_{i} \in[\mathbb{Z}]_{P, p_{\beta-2}, q_{\beta}}$ (if $\beta-1$ is not a limit) and $a_{i} \in[\mathbb{Z}]_{P, q_{\beta}}$ (if $\beta-1$ is a limit) and

(b) if $z=\sum a_{i} z_{i}$ with $z_{i}$ a root of a $\mathcal{G}\left(\Pi_{\beta-1}^{0}\right)$ component and $a_{i} \in[\mathbb{Z}]_{P}$, then $\mathcal{G} \models A_{\beta-1}(z) \wedge \Psi_{\beta-1}(z)$.

(3) For $\beta=\delta+2 \ell+3>3$ and $k \geq 0$,

(a) if $\mathcal{G}=u_{\beta-1, k}^{\infty} \mid z \wedge \Phi_{\beta-2}^{u_{\beta-1, k}}(z)$, then $z=\sum a_{i} z_{i}$ with $a_{i} \in[\mathbb{Z}]_{u_{\beta-1, k}, q_{\beta}, P}$ and $z_{i}$ a root of $a\left[\mathcal{G}\left(\Sigma_{\beta-2}^{0}\right)\right]_{u_{\beta-1, k}}$ component, and

(b) if $z=\sum a_{i} z_{i}$ with $a_{i} \in[\mathbb{Z}]_{u_{\beta-1, k}, P}$ and $z_{i}$ a root of a $\left[\mathcal{G}\left(\Sigma_{\beta-2}^{0}\right)\right]_{u_{\beta-1, k}}$ component, then $\mathcal{G} \models u_{\beta-1, k}^{\infty} \mid z \wedge \Phi_{\beta-2}^{u_{\beta-1, k}}(z)$.

(4) For $\beta=\delta+1$ and $k \geq 0$,

(a) If $\mathcal{G}=u_{\beta-1, k}^{\infty} \mid z \wedge \Phi_{f_{\beta-1}(k)}^{u_{\beta-1, k}}(z)$, then $z=\sum a_{i} z_{i}$ with $a_{i} \in[\mathbb{Z}]_{u_{\beta-1, k}, q_{\beta}, P}$ and $z_{i}$ a root of $a\left[\mathcal{G}\left(\Sigma_{f_{\beta-1}(k)}^{0}\right)\right]_{u_{\beta-1, k}}$ component, and

(b) if $z=\sum a_{i} z_{i}$ with $a_{i} \in[\mathbb{Z}]_{u_{\beta-1, k}, P}$ and $z_{i}$ a root of $a\left[\mathcal{G}\left(\sum_{f_{\beta-1}(k)}^{0}\right)\right]_{u_{\beta-1, k}}$ component, then $\mathcal{G} \models u_{\beta-1, k}^{\infty} \mid z \wedge \Phi_{f_{\beta-1}(k)}^{u_{\beta-1, k}}(z)$.

Before proving Lemma 4.13, we establish some notation and some basic facts which will be useful in the proof. Recall that $R_{\mathcal{G}}$ denotes the (distinct) set of roots of the recursively nested components of $\mathcal{G}$. $R_{\mathcal{G}}$ is a basis for $\mathcal{G}$ and for $D(\mathcal{G})$. Therefore, we can write any element of $\mathcal{G}$, as an element of $D(\mathcal{G})$, in the form $\sum q_{i} x_{i}$ where each $q_{i} \in \mathbb{Q}$ and $x_{i}$ is a root of a (nested) component of $\mathcal{G}$. We will often use various divisibility conditions to narrow which roots $x_{i}$ can occur in such a sum for particular elements and then use Lemmas 4.4 and 4.5 to restrict the possible values for the coefficients $q_{i}$.

Definition 4.14. If $X \subseteq R_{\mathcal{G}}$, then $\operatorname{Span}_{\mathcal{G}}(X)$ is the set of all element $g \in \mathcal{G}$ such that, in $D(\mathcal{G}), g=\sum q_{i} x_{i}$ where each $q_{i} \in \mathbb{Q}$ and $x_{i} \in X$.

Lemma 4.15. For any $X \subseteq R_{\mathcal{G}}, \operatorname{Span}_{\mathcal{G}}(X)$ is a pure subgroup of $\mathcal{G}$.

Proof. $\operatorname{Span}_{\mathcal{G}}(X)$ is clearly a subgroup. To see that it is pure, fix $g \in \operatorname{Span}_{\mathcal{G}}(X)$, $n>0$ and $h \in \mathcal{G}$ such that $n h=g$ in $\mathcal{G}$. We need to show that $h \in \operatorname{Span}_{\mathcal{G}}(X)$. Write $g=\sum q_{i} x_{i}$ (in $D(\mathcal{G})$ ) with $q_{i} \in \mathbb{Q}$ and $x_{i} \in X$. Because $\mathcal{G}$ is torsion-free, $h$ 
is the unique element satisfying $n h=g$. Therefore, in $D(\mathcal{G}), h=\sum\left(q_{i} / n\right) x_{i}$ and hence $h \in \operatorname{Span}_{\mathcal{G}}(X)$.

Note that in the context of torsion free abelian groups, the subgroup $\operatorname{Span}_{\mathcal{G}}(X)$ need not separate as a direct summand of $\mathcal{G}$. Nevertheless, in the proof of Lemma 4.13, we will often be able to describe the isomorphism types of such subgroups. The next lemma pertains to any torsion-free abelian group.

Lemma 4.16. Let $\mathcal{H}$ be a torsion-free abelian group which is $P$-closed for a set $P$ of primes. Let $\rho$ be a prime and let $h \in \mathcal{H}$ be infinitely divisible by $\rho$. Then for any $q \in[\mathbb{Z}]_{P}, q h$ is infinitely divisible by $\rho$.

Proof. Let $g \in \mathcal{H}$ satisfy $\rho^{k} g=h$. Since $\mathcal{H}$ is $[\mathbb{Z}]_{P}$-closed and $q \in[\mathbb{Z}]_{P}$, we can multiply this equation by $q$ in $\mathcal{H}$ to obtain $\left(q \rho^{k}\right) g=q h$. Thus, $q g$ witnesses that $q h$ is divisibly by $\rho^{k}$.

We return to the proof of Lemma 4.13. We work both within $\mathcal{G}$ and $D(\mathcal{G})$ during this proof and often rely on context to determine which group we are working in.

Proof of Lemma 4.13. Before establishing Lemma 4.13, we say a word about its proof. For $\beta=3$, we demonstrate (1) directly. For $\beta>3$, we demonstrate (2), (3), and (4) by simultaneous induction on $\beta$. The base case of the induction is the case $\beta=5$ for (3). The induction cases proceed as follows. To prove (2) for $\beta$, we use that (3) and (4) hold for values less than or equal to $\beta$; to prove (3) for $\beta$, we use that (2) holds for values less than $\beta$; and to prove (4) for $\beta$, we use that (3) holds for values less than $\beta$. Because (3) includes our base case, we begin with the proof of (3) after showing (1).

(1) For $\beta=3$, we show $y$ can be so expressed if $\mathcal{G} \models \Psi_{2}(y)$, i.e., if $\mathcal{G} \models p_{1}^{\infty} \mid y$. Working in $D(\mathcal{G})$, we express $y$ as $y=\sum a_{i} y_{i}$ where $a_{i} \in \mathbb{Q}$ and $y_{i}$ is the root of a $\mathcal{G}\left(\Sigma_{2}^{0}(m)\right)$ component, a $\mathcal{G}\left(\Pi_{2}^{0}\right)$ component, or a $[\mathbb{Z}]_{p_{3}}$ component. We note that it is impossible that any $y_{i}$ is the root of a $\mathcal{G}\left(\Sigma_{2}^{0}(m)\right)$ component. For if one were, with $y_{j}$ the root of a $\mathcal{G}\left(\Sigma_{2}^{0}\left(m_{j}\right)\right)$ component, there would be a $[\mathbb{Z}]_{p_{1}}$-multiple $\hat{y}$ of $y$ in $\mathcal{G}$ with

$$
\hat{y}=\sum_{i} \frac{\hat{a}_{i}}{p_{1}^{n_{i}}} y_{i}
$$

where $\hat{a}_{i} \in \mathbb{Z}, \hat{a}_{i} \neq 0$ implies $p_{1} \backslash \hat{a}_{i}$, and $n_{j}>m_{j}$. Since the coefficient of the root of any $\mathcal{G}\left(\Sigma_{2}^{0}\left(m_{j}\right)\right)$ component in $\mathcal{G}$ has the form $a / p_{1}^{k}$ where $a \in[\mathbb{Z}]_{q_{3}, P}$ and $k \leq m_{j}$, it must be that $\hat{a}_{j}=0$ and so $a_{j}=0$.

Thus, we have that $y=\sum a_{i} y_{i}$ where each $y_{i}$ is the root of a $[\mathbb{Z}]_{p_{3}}$ component or a $\mathcal{G}\left(\Pi_{2}^{0}\right)$ component. In other words, $y \in \mathcal{B}$ where $\mathcal{B}=\operatorname{Span}_{\mathcal{G}}(X)$ and $X$ is the set of roots of $\mathcal{G}\left(\Pi_{2}^{0}\right)$ components and $[\mathbb{Z}]_{p_{3}}$ components. $\mathcal{B}$ can be written as a direct sum of subgroups

$$
\left[\left\langle[\mathbb{Z}]_{p_{3}} \oplus \oplus_{k \in \omega}[\mathbb{Z}]_{p_{1}} ; q_{3}^{-t}\left(r+r_{k}\right): k, t \in \omega\right\rangle\right]_{P}
$$

since $\mathcal{G}\left(\Pi_{2}^{0}\right)=[\mathbb{Z}]_{p_{1}}$. Since $\mathcal{G} \models p_{1}^{\infty} \mid y$ and $\mathcal{B}$ is a pure subgroup of $\mathcal{G}$ (by Lemma 4.15), we have that $\mathcal{B} \models p_{1}^{\infty} \mid y$. Applying Lemma 4.4(2) to $\mathcal{B}$ (with $F_{1}=\left\{p_{3}\right\}$, $F_{2}=\left\{p_{1}\right\}, \rho=q_{3}$ and $P=P$ ) yields that each $y_{i}$ is the root of a $\mathcal{G}\left(\Pi_{2}^{0}\right)$ component and each $a_{i} \in[\mathbb{Z}]_{p_{1}, q_{3}, P}$. 
Conversely, suppose $y=\sum a_{i} y_{i}$ with $a_{i} \in[\mathbb{Z}]_{p_{1}, q_{3}, P}$ and $y_{i}$ the root of a $\mathcal{G}\left(\Pi_{2}^{0}\right)$ component. Since $y$ is the sum of roots of $\mathcal{G}\left(\Pi_{2}^{0}\right)$ components, $y \in \mathcal{B}$. Since each $a_{i} \in[\mathbb{Z}]_{p_{1}, q_{3}, P}$, Lemma 4.4(2) implies that $p_{1}^{\infty} \mid y$.

(3) For the base case when $\beta=5$, we first show (3)(a). Fix $k \in \omega$ and suppose that $\mathcal{G} \models u_{4, k}^{\infty} \mid z \wedge \Phi_{3}^{u_{4, k}}(z)$, recalling this is

$$
u_{4, k}^{\infty}\left|z \wedge p_{3}^{\infty}\right| z \wedge(\exists y)\left[u_{4, k}^{\infty}\left|y \wedge q_{3}^{\infty}\right|(z+y) \wedge p_{1}^{\infty} \mid y\right] .
$$

We need to show that $z=\sum a_{i} z_{i}$ with each $a_{i} \in[\mathbb{Z}]_{u_{4, k}, q_{5}, P}$ and each $z_{i}$ a root of a $\left[\mathcal{G}\left(\Sigma_{3}^{0}\right)\right]_{u_{4, k}}$ component.

Since $u_{4, k}^{\infty} \mid z$, the element $z$ must be a sum $z=\sum w_{i}$, where each $w_{i}$ comes from a $\left[\mathcal{G}\left(\Sigma_{3}^{0}\right)\right]_{u_{4, k}}$ or $\left[\mathcal{G}\left(\Pi_{3}^{0}\right)\right]_{u_{4, k}}$ component (which we denote by $\mathcal{G}_{i}$ ). Indeed, since $p_{3}^{\infty} \mid z$ by hypothesis, each $w_{i}$ is a multiple of the root of $\mathcal{G}_{i}$. Hence, the element $z$ must be a sum $z=\sum a_{i} z_{i}$ where $a_{i} \in \mathbb{Q}$ and each $z_{i}$ is the root of $\mathcal{G}_{i}$. We endeavor to show that, in fact, each $a_{i} \in[\mathbb{Z}]_{u_{4, k}, q_{5}, P}$ and each $\mathcal{G}_{i}$ is a $\left[\mathcal{G}\left(\Sigma_{3}^{0}\right)\right]_{u_{4, k}}$ component.

Fix a witness $y$ for $(\ddagger)$. Since $u_{4, k}^{\infty} \mid y, y$ must also be contained within the $\left[\mathcal{G}\left(\Sigma_{3}^{0}\right)\right]_{u_{4, k}}$ and $\left[\mathcal{G}\left(\Pi_{3}^{0}\right)\right]_{u_{4, k}}$ components. Furthermore, since $p_{1}^{\infty} \mid y, y$ must have the form $y=\sum b_{j} y_{j}$ where each $b_{j} \in \mathbb{Q}$ and $y_{j}$ is the root of a $\mathcal{G}\left(\Pi_{2}^{0}\right)$ component. Since the $\left[\mathcal{G}\left(\Pi_{3}^{0}\right)\right]_{u_{4, k}}$ components do not contain $\mathcal{G}\left(\Pi_{2}^{0}\right)$ components, each $y_{i}$ is the root of a $\mathcal{G}\left(\Pi_{2}^{0}\right)$ subcomponent of a $\left[\mathcal{G}\left(\Sigma_{3}^{0}\right)\right]_{u_{4, k}}$ component. Thus $z, y \in \mathcal{B}$ where $\mathcal{B}=\operatorname{Span}_{\mathcal{G}}(X)$ and $X$ contains the roots of the $\left[\mathcal{G}\left(\Pi_{3}^{0}\right)\right]_{u_{4, k}}$ components, the roots of the $\left[\mathcal{G}\left(\Sigma_{3}^{0}\right)\right]_{u_{4, k}}$ components and the roots of the $\mathcal{G}\left(\Pi_{2}^{0}\right)$ components of the $\left[\mathcal{G}\left(\Sigma_{3}^{0}\right)\right]_{u_{4, k}}$ components. By Lemma $4.15, \mathcal{B}$ is a pure subgroup of $\mathcal{G}$.

To describe the isomorphism type of $\mathcal{B}$, we need to analyze which primes infinitely divide the roots occurring in $X$. The point is that a particular element of $X$ may be the root of components at more than one level and each level will introduce different infinite divisibilities. Because of these considerations, we split into cases depending on whether $k>0$ or $k=0$.

First, consider the case when $k>0$ and let $r$ be the root of a $\left[\mathcal{G}\left(\Pi_{3}^{0}\right)\right]_{u_{4, k}}$ component or a $\left[\mathcal{G}\left(\Sigma_{3}^{0}\right)\right]_{u_{4, k}}$ component. The root $r$ is infinitely divisible by $p_{3}$ (since it is a root at level 3 ), by $u_{4, k}$ (by the prime closure of the component added at level 4 ) and by all the primes in $P$ (by the prime closure of $\mathcal{G}$ ). Because $k>0$, $r$ is not the root of a component at level 4 and because the level 3 (at which $r$ is a root) is odd, $r$ is not the root at a lower level. Similarly, if $r$ is the root of a $\mathcal{G}\left(\Pi_{2}^{0}\right)$ subcomponent of a $\left[\mathcal{G}\left(\Sigma_{3}^{0}\right)\right]_{u_{4, k}}$ component, then $r$ is infinitely divisible by $p_{1}$ (since it is the root of $\mathcal{G}\left(\Pi_{2}^{0}\right)$ ), by $u_{4, k}$ (by the prime closure) and by all the primes in $P$. Again, $r$ is not the root at any other level. Thus, when $k>0, \mathcal{B}$ is isomorphic to a direct sum of infinitely many copies of $[\mathbb{Z}]_{p_{3}, u_{4, k}, P}$ (coming from the roots of the $\left[\mathcal{G}\left(\Pi_{3}^{0}\right)\right]_{u_{4, k}}$ components) and infinitely many copies of

$$
\left[\left\langle[\mathbb{Z}]_{p_{3}} \oplus \oplus_{k \in \omega}[\mathbb{Z}]_{p_{1}} ; q_{3}^{-t}\left(r+r_{k}\right): k, t \in \omega\right\rangle\right]_{u_{4, k}, P}
$$

(coming from the roots of the $\left[\mathcal{G}\left(\Sigma_{3}^{0}\right)\right]_{u_{4, k}}$ components and the roots of their $\mathcal{G}\left(\Pi_{2}^{0}\right)$ subcomponents).

We show that each $z_{i}$ in the sum $z=\sum a_{i} z_{i}$ is the root of a $\left[\mathcal{G}\left(\Sigma_{3}^{0}\right)\right]_{u_{4, k}}$ component. If not, then we can suppose without loss of generality that $z_{0}$ is the root of a $\left[\mathcal{G}\left(\Pi_{3}^{0}\right)\right]_{u_{4, k}}$ component. That is, $z_{0}$ is the element 1 in a direct summand of $\mathcal{B}$ 
of the form $[\mathbb{Z}]_{p_{3}, u_{4, k}, P}$. Since $q_{3}^{\infty} \mid\left(\sum a_{i} z_{i}+\sum b_{j} y_{j}\right)$, there is a $[\mathbb{Z}]_{q_{3}}$ multiple $w$ of $z+y$ such that

$$
w=\sum \frac{n_{i}}{m_{i} q_{3}^{k_{i}}} z_{i}+\sum \frac{\widehat{n}_{j}}{\widehat{m}_{j} q_{3}^{l_{j}}} y_{j}
$$

where $q_{3} \backslash n_{i}, k_{i}>0, q_{3} \backslash \widehat{n}_{j}$ and $l_{j}>0$ (assuming $n_{i}, \widehat{n}_{j} \neq 0$ ). However, the coefficient of a multiple of $z_{0}$ in $\mathcal{B}$ must be from $[\mathbb{Z}]_{p_{3}, u_{4, k}, P}$. Hence, $n_{0}=0$ and therefore $a_{0}=0$.

Having established that each $z_{i}$ is the root of a $\left[\mathcal{G}\left(\Sigma_{3}^{0}\right)\right]_{u_{4, k}}$ component, it follows that $z, y \in \mathcal{B}^{\prime}$ where $\mathcal{B}^{\prime}=\operatorname{Span}_{\mathcal{G}}\left(X^{\prime}\right)$ with $X^{\prime} \subseteq X$ containing only the roots of the $\left[\mathcal{G}\left(\Sigma_{3}^{0}\right)\right]_{u_{4, k}}$ components and the roots of their $\mathcal{G}\left(\Pi_{2}^{0}\right)$ subcomponents. That is, $\mathcal{B}^{\prime}$ is the subgroup of $\mathcal{B}$ consisting of the direct sum of the infinitely many copies of the group in (2). Since $\mathcal{B}^{\prime}$ is a pure subgroup of $\mathcal{G}$, we have by Lemma 4.11(1)

$$
\mathcal{B}^{\prime} \models p_{3}^{\infty} \mid z \wedge(\exists y)\left[q_{3}^{\infty}\left|(z+y) \wedge p_{1}^{\infty}\right| y\right]
$$

(with our fixed element $y \in \mathcal{B}^{\prime}$ as witness). Therefore, we can apply Lemma 4.4(5) (with $F_{1}=\left\{p_{3}\right\}, F_{2}=\left\{p_{1}\right\}, \rho=q_{3}$ and $P=P \cup\left\{u_{4, k}\right\}$ ) to conclude that $z=\sum a_{i} z_{i}$ with $a_{i} \in[\mathbb{Z}]_{u_{4, k}, P}$.

Second, consider the case when $k=0$. In this case, $z=\sum a_{i} z_{i}$ where each $z_{i}$ is the root of a $\left[\mathcal{G}\left(\Sigma_{3}^{0}\right)\right]_{u_{4,0}}$ component since there are no $\left[\mathcal{G}\left(\Pi_{3}^{0}\right)\right]_{u_{4,0}}$ components. A root $r$ of a $\left[\mathcal{G}\left(\Sigma_{3}^{0}\right)\right]_{u_{4,0}}$ component is infinitely divisible by $p_{3}$ (since it is a root at level 3), by $u_{4, k}$ (by the prime closure), by $p_{4}$ (since $k=0$ and hence $r$ is also the root of a $\mathcal{G}\left(\Pi_{4}^{0}\right)$ or $\mathcal{G}\left(\Sigma_{4}^{0}(m)\right)$ component) and by all the primes in $P$. Furthermore, if $r, r^{\prime}$ are roots of (distinct) $\mathcal{G}\left(\Pi_{4}^{0}\right)$ or $\mathcal{G}\left(\Sigma_{4}^{0}(m)\right)$ components within the same copy of $\mathcal{G}\left(\Pi_{5}^{0}\right)$ or $\mathcal{G}\left(\Sigma_{5}^{0}\right)$, then $r-r^{\prime}$ is infinitely divisible by $q_{5}$. (This divisibility does not add to the infinite divisibility of either $r$ or $r^{\prime}$, but it does effect the isomorphism type of $\mathcal{B}$.) However, if $r, r^{\prime}$ are roots of such components in different copies of $\mathcal{G}\left(\Pi_{5}^{0}\right)$ or $\mathcal{G}\left(\Sigma_{5}^{0}\right)$, then $r-r^{\prime}$ is not divisible by $q_{5}$. To smooth out this difference in divisibility and to simplify the calculations, we work in $[\mathcal{B}]_{q_{5}}$.

The group $[\mathcal{B}]_{q_{5}}$ is isomorphic to the direct sum of infinite many copies of

$$
\left[\left\langle[\mathbb{Z}]_{p_{3}, p_{4}} \oplus \oplus_{k \in \omega}[\mathbb{Z}]_{p_{1}} ; q_{3}^{-t}\left(r+r_{k}\right): k, t \in \omega\right\rangle\right]_{u_{4,0}, q_{5}, P}
$$

(coming from the roots of the $\left[\mathcal{G}\left(\Sigma_{3}^{0}\right)\right]_{u_{4,0}}$ components and their $\mathcal{G}\left(\Pi_{2}^{0}\right)$ subcomponents). Since $\mathcal{B}$ is a pure subgroup of $\mathcal{G}$ and since $[\mathcal{B}]_{q_{5}}$ is an expansion of $\mathcal{B}$, we have by Lemma 4.11(1) and 4.11(2)

$$
[\mathcal{B}]_{q_{5}} \models p_{3}^{\infty} \mid z \wedge(\exists y)\left[q_{3}^{\infty}\left|(z+y) \wedge p_{1}^{\infty}\right| y\right]
$$

with our fixed element $y \in B$ as the witness. Applying Lemma 4.4(5) with $F_{1}=$ $\left\{p_{3}, p_{4}\right\}, F_{2}=\left\{p_{1}\right\}, \rho=q_{3}$ and $P=P \cup\left\{u_{4,0}, q_{5}\right\}$, we conclude that $a_{i} \in[\mathbb{Z}]_{P, u_{4,0}, q_{5}}$. This completes the proof of (3)(a) when $\beta=5$.

To prove (3)(b) when $\beta=5$, assume $z=\sum a_{i} z_{i}$, where $a_{i} \in[\mathbb{Z}]_{u_{4, k}, P}$ and each $z_{i}$ is the root of a $\left[\mathcal{G}\left(\Sigma_{3}^{0}\right)\right]_{u_{4, k}}$ component. Let $\mathcal{G}_{i}$ denote the $\left[\mathcal{G}\left(\Sigma_{3}^{0}\right)\right]_{u_{4, k}}$ component containing $z_{i}$. We need to show that

$$
u_{4, k}^{\infty}\left|z \wedge p_{3}^{\infty}\right| z \wedge(\exists y)\left[u_{4, k}^{\infty}\left|y \wedge q_{3}^{\infty}\right|(z+y) \wedge p_{1}^{\infty} \mid y\right] .
$$

Since $z_{i}$ is the root of $\mathcal{G}_{i}$, we have $p_{3}^{\infty} \mid z_{i}$. By Lemma 4.16 and the fact that $\mathcal{G}_{i}$ is $P \cup\left\{u_{4, k}\right\}$-closed, it follows that $p_{3}^{\infty} \mid a_{i} z_{i}$ and $u_{4, k}^{\infty} \mid a_{i} z_{i}$. Hence, $p_{3}^{\infty} \mid z$ and $u_{4, k}^{\infty} \mid z$.

Let $y_{i}$ be the root of a $\mathcal{G}\left(\Pi_{2}^{0}\right)$ component inside $\mathcal{G}_{i}$ and let $y=\sum a_{i} y_{i}$. Since $\mathcal{G}\left(\Pi_{2}^{0}\right) \cong[\mathbb{Z}]_{p_{1}}$, we have $p_{1}^{\infty} \mid y_{i}$. As $\mathcal{G}_{i}$ is $P \cup\left\{u_{4, k}\right\}$-closed, it follows that $p_{1}^{\infty} \mid a_{i} y_{i}$ 
(by Lemma 4.16) and that $u_{4, k}^{\infty} \mid a_{i} y_{i}$. Hence, $p_{1}$ and $u_{4, k}$ infinitely divide $y$. By the definition of $\mathcal{G}\left(\Sigma_{3}^{0}\right), q_{3}^{\infty} \mid\left(z_{i}+y_{i}\right)$ and applying Lemma 4.16 one more time, $q_{3}^{\infty} \mid\left(a_{i} z_{i}+a_{i} y_{i}\right)$. Therefore $\mathcal{G}$ satisfies $\Phi_{3}^{u_{4, k}}(z)$ with witness $y$.

This completes the base case of $\beta=5$.

Next, we show (3) for $\beta>5$ supposing (2) holds for $\beta-2$. To prove (3)(a), we suppose $\mathcal{G} \models u_{\beta-1, k}^{\infty} \mid z \wedge \Phi_{\beta-2}^{u_{\beta-1, k}}(z)$, recalling this is

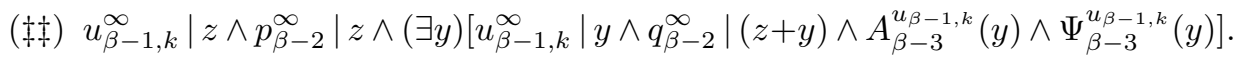

We need to show that $z=\sum a_{i} z_{i}$ with each $a_{i} \in[\mathbb{Z}]_{u_{\beta-1, k}, q_{\beta}, P}$ and each $z_{i}$ a root of a $\left[\mathcal{G}\left(\Sigma_{\beta-2}^{0}\right)\right]_{u_{\beta-1, k}}$ component.

As in the $\beta=5$ case, $u_{\beta-1, k}^{\infty} \mid z$ and $p_{\beta-2}^{\infty} \mid z$ imply that $z=\sum a_{i} z_{i}$, where $a_{i} \in \mathbb{Q}$ and each $z_{i}$ is the root of a $\left[\mathcal{G}\left(\Sigma_{\beta-2}^{0}\right)\right]_{u_{\beta-1, k}}$ or $\left[\mathcal{G}\left(\Pi_{\beta-2}^{0}\right)\right]_{u_{\beta-1, k}}$ component. We endeavor to show that, in fact, each $a_{i} \in[\mathbb{Z}]_{u_{\beta-1, k}, q_{\beta}, P}$ and each $z_{i}$ is the root of a $\left[\mathcal{G}\left(\Sigma_{\beta-2}^{0}\right)\right]_{u_{\beta-1, k}}$ component.

Fix a witness $y$ for ( $\ddagger)$. Our first goal is to show that $y$ is a sum of roots of $\mathcal{G}\left(\Pi_{\beta-3}^{0}\right)$ components. Since $u_{\beta-1, k}^{\infty} \mid y, y$ lies within the $\left[\mathcal{G}\left(\Sigma_{\beta-2}^{0}\right)\right]_{u_{\beta-1, k}}$ and $\left[\mathcal{G}\left(\Pi_{\beta-2}^{0}\right)\right]_{u_{\beta-1, k}}$ components. Thus, $y \in \mathcal{H}=\operatorname{Span}_{\mathcal{G}}(X)$ where $X$ contains the roots of the $\left[\mathcal{G}\left(\Sigma_{\beta-2}^{0}\right)\right]_{u_{\beta-1, k}}$ and $\left[\mathcal{G}\left(\Pi_{\beta-2}^{0}\right)\right]_{u_{\beta-1, k}}$ components as well as the roots of all the components nested via the recursive construction inside these components. Note that $\mathcal{H}$ is the subgroup of $\mathcal{G}$ consisting of the elements infinitely divisible by $u_{\beta-1, k}$. Because $\mathcal{G}$ satisfies $A_{\beta-3}^{u_{\beta-1, k}}(y) \wedge \Psi_{\beta-3}^{u_{\beta-1, k}}(y)$, we have that $\mathcal{H}$ satisfies $A_{\beta-3}(y) \wedge \Psi_{\beta-3}(y)$ by Lemma 4.6.

We describe the isomorphism type of $\mathcal{H}$ in two cases: when $k>0$ and when $k=0$. If $k>0$, then the roots of the $\left[\mathcal{G}\left(\Sigma_{\beta-2}^{0}\right)\right]_{u_{\beta-1, k}}$ and $\left[\mathcal{G}\left(\Pi_{\beta-2}^{0}\right)\right]_{u_{\beta-1, k}}$ components are not roots of components at any other levels. Thus, $\mathcal{H}$ is an infinite direct sum of $\left[\mathcal{G}\left(\Sigma_{\beta-2}^{0}\right)\right]_{u_{\beta-1, k}, P}$ and $\left[\mathcal{G}\left(\Pi_{\beta-2}^{0}\right)\right]_{u_{\beta-1, k}, P}$ groups.

If $k=0$, then note that there are no $\left[\mathcal{G}\left(\Pi_{\beta-2}^{0}\right)\right]_{u_{\beta-1,0}}$ components. Each root of a $\left[\mathcal{G}\left(\Sigma_{\beta-2}^{0}\right)\right]_{u_{\beta-1,0}}$ component is also the root of a $\mathcal{G}\left(\Sigma_{\beta-1}^{0}(m)\right)$ or a $\mathcal{G}\left(\Pi_{\beta-1}^{0}\right)$ component. Thus, each such root is infinitely divisible by $p_{\beta-1}$ (in addition to the divisibility imposed at level $\beta-2)$. Furthermore, if $r, r^{\prime}$ are roots of $\mathcal{G}\left(\Sigma_{\beta-1}^{0}(m)\right)$ or $\mathcal{G}\left(\Pi_{\beta-1}^{0}\right)$ components from the same copy of $\mathcal{G}\left(\Sigma_{\beta}^{0}\right)$ or $\mathcal{G}\left(\Pi_{\beta}^{0}\right)$, then $q_{\beta}^{\infty} \mid\left(r-r^{\prime}\right)$. If they are roots from different copies of $\mathcal{G}\left(\Sigma_{\beta}^{0}\right)$ or $\mathcal{G}\left(\Pi_{\beta}^{0}\right)$, then we have no such $q_{\beta}$ divisibility. To incorporate the extra divisibility by $p_{\beta-1}$ and to smooth out this divisibility difference, we look at $[\mathcal{H}]_{q_{\beta}, p_{\beta-1}}$. $[\mathcal{H}]_{q_{\beta}, p_{\beta-1}}$ is isomorphic to an infinite direct sum of $\left[\mathcal{G}\left(\Sigma_{\beta-2}^{0}\right)\right]_{P^{\prime}}$ groups where $P^{\prime}=P \cup\left\{u_{\beta-1,0}, q_{\beta}, p_{\beta-1}\right\}$.

In each of the $k>0$ and $k=0$ cases, we can apply Part $(2)\left(\right.$ a) for $\mathcal{H}$ or $[\mathcal{H}]_{q_{\beta}, p_{\beta-1}}$ and $\beta-2$ to conclude that $y=\sum b_{j} y_{j}$ is a sum of roots $y_{j}$ of $\mathcal{G}\left(\Pi_{\beta-3}^{0}\right)$ components in $\mathcal{G}$ (with appropriate coefficients, which depend on which case we are in). Thus, we have established our first goal.

Our second goal is to show that in the sum $z=\sum a_{i} z_{i}$, each $z_{i}$ is the root of a $\left[\mathcal{G}\left(\Sigma_{\beta-2}^{0}\right)\right]_{u_{\beta-1, k}}$ component as opposed to a $\left[\mathcal{G}\left(\Pi_{\beta-2}^{0}\right)\right]_{u_{\beta-1, k}}$ component and each coefficient $a_{i}$ lies in $[\mathbb{Z}]_{p_{\beta-1}, q_{\beta}, P}$. We have $z, y \in \mathcal{B}=\operatorname{Span}_{\mathcal{G}}(X)$ where $X$ contains the roots of the $\left[\mathcal{G}\left(\Pi_{\beta-2}^{0}\right)\right]_{u_{\beta-1, k}}$ components, the roots of the $\left[\mathcal{G}\left(\Sigma_{\beta-2}^{0}\right)\right]_{u_{\beta-1, k}}$ components and the roots of their $\mathcal{G}\left(\Pi_{\beta-3}^{0}\right)$ components. We split into cases depending on whether $k>0$ or $k=0$ and proceed with an analysis of the infinite divisibilities as in the $\beta=5$ case. 
First, suppose that $k>0$. A root $r$ of a $\left[\mathcal{G}\left(\Pi_{\beta-2}^{0}\right)\right]_{u_{\beta-1, k}}$ or $\left[\mathcal{G}\left(\Sigma_{\beta-2}^{0}\right)\right]_{u_{\beta-1, k}}$ component is infinitely divisible by $p_{\beta-2}$ (being a root at level $\beta-2$ ) and by $u_{\beta-1, k}$ and the primes in $P$ (by prime closures). Since $\beta-2$ is odd, $r$ is not a root at a lower level, and since $k>0, r$ is not a root at a higher level.

A root $r$ of a $\mathcal{G}\left(\Pi_{\beta-3}^{0}\right)$ subcomponent of a $\left[\mathcal{G}\left(\Sigma_{\beta-2}^{0}\right)\right]_{u_{\beta-1, k}}$ component is infinitely divisible by $p_{\beta-3}$ (being a root at level $\beta-3$ ) and by $u_{\beta-1, k}$ and the primes in $P$ (by divisible closures). In addition, if $\beta-3$ is not a limit ordinal, then $r$ is also the root of a $\left[\mathcal{G}\left(\Sigma_{\beta-4}^{0}\right)\right]_{u_{\beta-3,0}}$ component and hence is infinitely divisible by $p_{\beta-4}$ and $u_{\beta-3,0}$. Notice that the recursion stops at this point because $\beta-4$ is an odd ordinal and hence $r$ is not the root at any lower level. If $\beta-3$ is a limit ordinal, then $r$ is also the root of a $\left[\mathcal{G}\left(\Sigma_{f_{\beta-3}(0)}^{0}\right)\right]_{u_{\beta-3,0}}$ component and hence is infinitely divisible by $p_{f_{\beta-3}(0)}$ and $u_{\beta-3,0}$. Again, the recursion stops at this point because $f_{\beta-3}(0)$ is an odd ordinal. Recall that if $\beta-3$ is not a limit, then $f_{\beta-3}(0)=\beta-4$. Thus, we can also describe the infinite divisibility by $p_{\beta-4}$ (in the case when $\beta-3$ is not a limit) as infinite divisibility by $p_{f_{\beta-3}(0)}$. In future analyses, we will combine these cases in this manner.

From this analysis, when $k>0$, the group $\mathcal{B}$ is isomorphic to the direct sum of infinitely many copies of $[\mathbb{Z}]_{p_{\beta-2}, u_{\beta-1, k}, P}$ (from the roots of $\left[\mathcal{G}\left(\Pi_{\beta-2}^{0}\right)\right]_{u_{\beta-1, k}}$ components) and infinitely many copies of

$$
\left[\left\langle[\mathbb{Z}]_{F_{1}} \oplus \bigoplus_{j \in \omega}[\mathbb{Z}]_{F_{2}} ; \frac{x+y_{j}}{\rho^{k}}: j, k \in \omega\right\rangle\right]_{P, u_{\beta-1, k}}
$$

where $F_{1}=\left\{p_{\beta-2}\right\}, F_{2}=\left\{p_{\beta-3}, u_{\beta-3,0}, p_{f_{\beta-3}(0)}\right\}$ and $\rho=q_{\beta-2}$ (from the roots of $\left[\mathcal{G}\left(\Sigma_{\beta-2}^{0}\right)\right]_{u_{\beta-1, k}}$ components and their $\mathcal{G}\left(\Pi_{\beta-3}^{0}\right)$ subcomponents). A divisibility argument almost identical to the one use in the $\beta=5$ case (using the fact that $\left.q_{\beta-2}^{\infty} \mid(z+y)\right)$ shows that none of the $z_{i}$ elements can come from the $[\mathbb{Z}]_{p_{\beta-2}, u_{\beta-1, k}, P}$ summands. Therefore, each $z_{i}$ is the root of a $\left[\mathcal{G}\left(\Sigma_{\beta-2}^{0}\right)\right]_{u_{\beta-1, k}}$ component.

Let $\mathcal{B}^{\prime}$ be the subgroup of $\mathcal{B}$ consisting of the direct sum of infinitely many copies of the group in Equation (3). Since $\mathcal{B}^{\prime}$ is a pure subgroup of $\mathcal{G}$ containing $y$ and $z$ and $\mathcal{G}$ satisfies

$$
p_{\beta-2}^{\infty}\left|z \wedge q_{\beta-2}^{\infty}\right|(z+y) \wedge p_{\beta-3}^{\infty} \mid y
$$

we have that this formula is also satisfied in $\mathcal{B}^{\prime}$ (by Lemma 4.11(1)). Applying Lemma 4.4(5) to $\mathcal{B}^{\prime}$ with the above values for $F_{1}, F_{2}$ and $\rho$ yields that each $a_{i} \in$ $[\mathbb{Z}]_{P, u_{\beta-1, k}}$, completing the case when $k>0$.

Second, suppose $k=0$. The analysis of the isomorphism type of $\mathcal{B}$ is almost identical to the case when $k>0$ except for three points. First, there are no components of the form $\left[\mathcal{G}\left(\Pi_{\beta-2}^{0}\right)\right]_{u_{\beta-1,0}}$ and hence no argument is needed to conclude that each $z_{i}$ is the root of a $\left[\mathcal{G}\left(\Sigma_{\beta-2}^{0}\right)\right]_{u_{\beta-1,0}}$ component. Second, the root of a $\left[\mathcal{G}\left(\Sigma_{\beta-2}^{0}\right)\right]_{u_{\beta-1,0}}$ component is also the root of a $\mathcal{G}\left(\Sigma_{\beta-1}^{0}\right)$ or $\mathcal{G}\left(\Pi_{\beta-1}^{0}\right)$ component and hence is infinitely divisible by $p_{\beta-1}$ in addition to the infinite divisibilities given above. Third, to smooth out the fact that $q_{\beta}$ infinitely divides $r-r^{\prime}$ when $r, r^{\prime}$ are roots of $\mathcal{G}\left(\Sigma_{\beta-1}^{0}\right)$ or $\mathcal{G}\left(\Pi_{\beta-1}^{0}\right)$ components from the same $\mathcal{G}\left(\Sigma_{\beta}^{0}\right)$ or $\mathcal{G}\left(\Pi_{\beta}^{0}\right)$ component, we work with $[\mathcal{B}]_{q_{\beta}}$. With these observations, $[\mathcal{B}]_{q_{\beta}}$ is isomorphic to the direct 
sum of infinitely many copies of

$$
\left[\left\langle[\mathbb{Z}]_{F_{1}} \oplus \bigoplus_{j \in \omega}[\mathbb{Z}]_{F_{2}} ; \frac{x+y_{j}}{\rho^{k}}: j, k \in \omega\right\rangle\right]_{P, u_{\beta-1, k}, q_{\beta}}
$$

where $F_{1}=\left\{p_{\beta-2}, p_{\beta-1}\right\}, F_{2}=\left\{p_{\beta-3}, u_{\beta-3,0}, p_{f_{\beta-3}(0)}\right\}$ and $\rho=q_{\beta-2}$. Since $\mathcal{B}$ is a pure subgroup of $\mathcal{G}$ and $\mathcal{G}$ satisfies

$$
p_{\beta-2}^{\infty}\left|z \wedge q_{\beta-2}^{\infty}\right|(z+y) \wedge p_{\beta-3}^{\infty} \mid y
$$

this formula is also satisfied in $\mathcal{B}$ (by Lemma $4.11(1)$ ). Since $[\mathcal{B}]_{q_{\beta}}$ is an expansion of $\mathcal{B}$, it remains true in $\mathcal{B}^{\prime}$ (by Lemma $4.11(2)$ ). We apply Lemma 4.4(5) to conclude that each $a_{i} \in[\mathbb{Z}]_{P, u_{\beta-1, k}, q_{\beta}}$.

To prove (3)(b) when $\beta>5$, fix an element $z=\sum a_{i} z_{i}$ with each $a_{i} \in[\mathbb{Z}]_{u_{\beta-1, k}, P}$ and each $z_{i}$ the root of a $\left[\mathcal{G}\left(\Sigma_{\beta-2}^{0}\right)\right]_{u_{\beta-1, k}}$ component (which we denote by $\mathcal{G}_{i}$ ). We have $u_{\beta-1}^{\infty} \mid z_{i}$ and $p_{\beta-2}^{\infty} \mid z_{i}$ as a consequence of the structure of $\left[\mathcal{G}\left(\Sigma_{\beta-2}^{0}\right)\right]_{u_{\beta-1, k}}$ components and $z_{i}$ being the root. As $a_{i} \in[\mathbb{Z}]_{u_{\beta-1, k}, P}$ and $\mathcal{G}_{i}$ is $\left\{u_{\beta-1, k}, P\right\}$-closed, it follows that $u_{\beta-1}^{\infty} \mid a_{i} z_{i}$ and $p_{\beta-2}^{\infty} \mid a_{i} z_{i}$ and hence that $u_{\beta-1}^{\infty} \mid z$ and $p_{\beta-2}^{\infty} \mid z$.

Let $y:=\sum a_{i} y_{i}$, where $y_{i}$ is the root of a $\mathcal{G}\left(\Pi_{\beta-3}^{0}\right)$ subcomponent of $\mathcal{G}_{i}$. Since each $\mathcal{G}_{i}$ is a $\left[\mathcal{G}\left(\Sigma_{\beta-2}^{0}\right)\right]_{u_{\beta-1, k}}$ component, it follows from the structure of these components that $u_{\beta-1, k}^{\infty} \mid y$ and $q_{\beta-2}^{\infty} \mid(z+y)$. It remains to show that $\mathcal{G}$ satisfies $A_{\beta-3}^{u_{\beta-1, k}}(y)$ and $\Psi_{\beta-3}^{u_{\beta-1, k}}(y)$.

Let $\mathcal{H}=\operatorname{Span}_{\mathcal{G}}(X)$ where $X$ contains the roots of the $\left[\mathcal{G}\left(\Sigma_{\beta-2}^{0}\right)\right]_{u_{\beta-1, k}}$ components and the roots of any component nested via the recursive construction inside such a component. Note that $y, z \in \mathcal{H}$ and that $\mathcal{H}$ is the subgroup of $\mathcal{G}$ consisting of the elements which are infinitely divisible by $u_{\beta-1, k}$. Applying Part (2)(b) to $\mathcal{H}$ with $\beta-2$ and $P=P \cup\left\{u_{\beta-1, k}\right\}$, we get that $\mathcal{H}$ satisfies $A_{\beta-3}(y) \wedge \Psi_{\beta-3}(y)$. Since $\mathcal{H}$ consists of the elements of $\mathcal{G}$ which are infinitely divisible by $u_{\beta-1, k}$, we have that $\mathcal{G}$ satisfies $A_{\beta-3}^{u_{\beta-1, k}}(y) \wedge \Psi_{\beta-3}^{u_{\beta-1, k}}(y)$ by Lemma 4.6 as required.

(2) We show (2) for $\beta$ supposing (3) and (4) hold for values less than or equal to $\beta$. To show (2)(a), we suppose $\mathcal{G} \models A_{\beta-1}(z) \wedge \Psi_{\beta-1}(z)$, recalling $A_{\beta-1}(z)$ is

$$
p_{\beta-1}^{\infty} \mid z \wedge(\exists w)\left[u_{\beta-1,1}\left|w \wedge v_{\beta-1,0}^{\infty}\right|(z+w)\right] .
$$

Since $p_{\beta-1}^{\infty} \mid z$, we can express $z$ as $z=\sum a_{i} z_{i}$ where $a_{i} \in \mathbb{Q}$ and $z_{i}$ is the root of a $\mathcal{G}\left(\Sigma_{\beta-1}^{0}(m)\right)$ or $\mathcal{G}\left(\Pi_{\beta-1}^{0}\right)$ component (which we denote by $\left.\mathcal{G}_{i}\right)$. Since $z_{i}$ is also the root of the $\left[\mathcal{G}\left(\Sigma_{f_{\beta-1}(0)}^{0}\right)\right]_{u_{\beta-1,0}}$ component inside this $\mathcal{G}\left(\Sigma_{\beta-1}^{0}(m)\right)$ or $\mathcal{G}\left(\Pi_{\beta-1}^{0}\right)$ component, we have that $z_{i}$ is infinitely divisible by $p_{f_{\beta-1}(0)}$ and $u_{\beta-1,0}$. As above, the recursion stops here since $f_{\beta-1}(0)$ is an odd ordinal and hence $z_{i}$ is the element 1 in a copy of $[\mathbb{Z}]_{p_{f_{\beta-1}(0)}}$.

Fix an element $w$ witnessing $\mathcal{G} \models A_{\beta-1}(z)$. The condition $u_{\beta-1,1}^{\infty} \mid w$ implies that $w$ is a sum of elements from $\left[\mathcal{G}\left(\Sigma_{f_{\beta-1}(1)}^{0}\right)\right]_{u_{\beta-1,1}}$ and $\left[\mathcal{G}\left(\Pi_{f_{\beta-1}(1)}^{0}\right)\right]_{u_{\beta-1,1}}$ components. The condition $v_{\beta-1,0}^{\infty} \mid(z+w)$ implies (by divisibility arguments similar to those already given many times) that $w=\sum b_{i} w_{i}$, where each $b_{i} \in \mathbb{Q}$ and each $w_{i}$ is the root of a $\left[\mathcal{G}\left(\Sigma_{f_{\beta-1}(1)}^{0}\right)\right]_{u_{\beta-1,1}}$ or $\left[\mathcal{G}\left(\Pi_{f_{\beta-1}(1)}^{0}\right)\right]_{u_{\beta-1,1}}$ component. Since $f_{\beta-1}(1)$ is an odd ordinal, the root of such a component is the element 1 in a copy of $[\mathbb{Z}]_{p_{f_{\beta-1}(1)}}$. Thus, $w_{i}$ is infinitely divisible by $p_{f_{\beta-1}(1)}$ and $u_{\beta-1,1}$ but the recursion stops at this point. (Note that for the same reason, the roots of $\left[\mathcal{G}\left(\Sigma_{f_{\beta-1}(k)}^{0}\right)\right]_{u_{\beta-1, k}}$ and 
$\left[\mathcal{G}\left(\Pi_{f_{\beta-1}(k)}^{0}\right)\right]_{u_{\beta-1, k}}$ components for $k \geq 2$ are infinitely divisible only by $p_{f_{\beta-1}(k)}$ and $u_{\beta-1, k}$.)

To find the coefficients $a_{i}$ in $z=\sum a_{i} z_{i}$, let $\mathcal{B}=\operatorname{Span}_{\mathcal{G}}(X)$ where $X$ contains the roots of the $\left[\mathcal{G}\left(\Sigma_{f_{\beta-1}(k)}^{0}\right)\right]_{u_{\beta-1, k}}$ and $\left[\mathcal{G}\left(\Pi_{f_{\beta-1}(k)}^{0}\right)\right]_{u_{\beta-1, k}}$ components for $k \in \omega$. As in the proof of Part (3), we work in $[\mathcal{B}]_{q_{\beta}}$ since, for roots $r, r^{\prime}$ of $\left[\mathcal{G}\left(\Sigma_{f_{\beta-1}(0)}^{0}\right)\right]_{u_{\beta-1,0}}$ components (which are also roots of $\mathcal{G}\left(\Sigma_{\beta-1}^{0}(m)\right)$ or $\mathcal{G}\left(\Pi_{\beta-1}^{0}\right)$ components), $q_{\beta}$ infinitely divides $r-r^{\prime}$ if and only if these roots come from the same $\mathcal{G}\left(\Sigma_{\beta}^{0}\right)$ or $\mathcal{G}\left(\Pi_{\beta}^{0}\right)$ summand of $\mathcal{G}$.

$[\mathcal{B}]_{q_{\beta}}$ is isomorphic to the $P^{\prime}=P \cup\left\{q_{\beta}\right\}$ closure of

$$
\left\langle\mathcal{F} ; \frac{x_{k, j}}{\sigma_{k}^{l}}, \frac{x_{k, j}+x_{k+1, j}}{\rho_{k}^{l}}: j, k, l \in \omega \text { and } \sigma_{k} \in F_{k}\right\rangle
$$

where $\mathcal{F}$ is the free abelian group on $x_{k, j}($ for $k, j \in \omega), F_{0}=\left\{p_{f_{\beta-1}(0)}, p_{\beta-1}, u_{\beta-1,0}\right\}$, $F_{k}=\left\{p_{f_{\beta-1}(k)}, u_{\beta-1, k}\right\}$ (for $k>0$ ) and $\rho_{k}=v_{\beta-1, k}$. (In this presentation, for each fixed $j, x_{k, j}$ is the root of a $\left[\mathcal{G}\left(\Sigma_{f_{\beta-1}(0)}^{0}\right)\right]_{u_{\beta-1, k}}$ component or a $\left[\mathcal{G}\left(\Pi_{f_{\beta-1}(0)}^{0}\right)\right]_{u_{\beta-1, k}}$ component within a fixed $\mathcal{G}\left(\Sigma_{\beta-1}^{0}(m)\right)$ or $\mathcal{G}\left(\Pi_{\beta-1}^{0}\right)$ component of $\mathcal{G}$. As $j$ varies, we range over all $\mathcal{G}\left(\Sigma_{\beta-1}^{0}(m)\right)$ and $\mathcal{G}\left(\Pi_{\beta-1}^{0}\right)$ components of $\mathcal{G}$.) If $\beta-1$ is a limit, then $p_{f_{\beta-1}(k)} \neq p_{f_{\beta-1}\left(k^{\prime}\right)}$ for $k \neq k^{\prime}$. If $\beta-1$ is not a limit, then each $p_{f_{\beta-1}(k)}=p_{\beta-2}$ for all $k$. In this case, we can remove the primes $p_{f_{\beta-1}(k)}$ from $F_{k}$ and add $p_{\beta-2}$ to $P^{\prime}$ (since $[\mathcal{B}]_{q_{\beta}}$ is $p_{\beta-2}$ closed). This change has the effect of including infinite divisibility by $p_{\beta-2}$ for our coefficients $a_{i}$.

Since $\mathcal{B}$ is a pure subgroup of $\mathcal{G}, \mathcal{B}$ is a subgroup of $[\mathcal{B}]_{q_{\beta}}$ and $z, w \in \mathcal{B}$, we have (applying both Lemma $4.11(1)$ and $4.11(2)$ ) that $[\mathcal{B}]_{q_{\beta}}$ satisfies $A_{\beta-1}(z)$ with our element $w$ are witness. Therefore, by Lemma 4.5(3), we obtain that $z=\sum a_{i} z_{i}$ with $a_{i} \in[\mathbb{Z}]_{P, p_{\beta-2}, q_{\beta}}$ (if $\beta-1$ is not a limit) or $a_{i} \in[\mathbb{Z}]_{P, q_{\beta}}$ (if $\beta-1$ is a limit).

Next, we use the fact that $\mathcal{G} \models \Psi_{\beta-1}(z)$ to show each $\mathcal{G}_{i}$ is a $\mathcal{G}\left(\Pi_{\beta-1}^{0}\right)$ component. For if one were not, then some $\mathcal{G}_{\ell}$ would be a $\mathcal{G}\left(\Sigma_{\beta-1}^{0}\left(m_{0}\right)\right)$ component for some $m_{0} \in \omega$. With $m:=m_{0}+1$, we fix a sequence $g_{0}, g_{1}, \ldots, g_{m}$ witnessing that $\mathcal{G}$ satisfies the $m$-th conjunct of $\Psi_{\beta-1}(z)$. Since $\mathcal{G}$ satisfies $u_{\beta-1, m}^{\infty} \mid g_{m}$ and $\Phi_{f_{\beta-1}(m)}^{u_{\beta-1, m}}\left(g_{m}\right)$, we have by Part (3) or Part (4) (depending on the form of $f_{\beta-1}(m)$ ) that $g_{m}=\sum c_{j} y_{j}$ where each $y_{j}$ is the root of a $\left[\mathcal{G}\left(\sum_{f_{\beta-1}(m)}^{0}\right)\right]_{u_{\beta-1, m}}$ component. Since $g_{0}=z=\sum a_{i} z_{i}$ and $v_{\beta-1}^{\infty} \mid\left(g_{k}+g_{k+1}\right)$ for $0 \leq k<m$, one of the $y_{j}$ roots in the summand for $g_{m}$ must lie in the component $\mathcal{G}_{l}$. However, $\mathcal{G}_{l}$ is a $\mathcal{G}\left(\Sigma_{\beta-1}^{0}\left(m_{0}\right)\right)$ component with $m_{0}<m$, so it does not contain a $\left[\mathcal{G}\left(\Sigma_{f_{\beta-1}(m)}^{0}\right)\right]_{u_{\beta-1, m}}$ component, yielding the desired contradiction. This completes the proof of (2)(a).

To prove (2)(b), fix an element $z=\sum a_{i} z_{i}$ with $a_{i} \in[\mathbb{Z}]_{P}$ and $z_{i}$ a root of a $\mathcal{G}\left(\Pi_{\beta-1}^{0}\right)$ component of $\mathcal{G}$ (which we denote $\mathcal{G}_{i}$ ). We need to show that $\mathcal{G} \models A_{\beta-1}(z)$ and $\mathcal{G} \models \Psi_{\beta-1}(z)$. For the former, we need to show that $\mathcal{G}$ satisfies

$$
p_{\beta-1}^{\infty} \mid z \wedge(\exists w)\left[u_{\beta-1,1}^{\infty}\left|w \wedge v_{\beta-1,0}^{\infty}\right|(z+w)\right] .
$$

From the structure of $\mathcal{G}\left(\Pi_{\beta-1}^{0}\right)$ components, we have each $z_{i}$ is the root $r_{0}$ of the $\left[\mathcal{G}\left(\Sigma_{f_{\beta-1}(0)}^{0}\right)\right]_{u_{\beta-1,0}}$ component of $\mathcal{G}_{i}$. The condition $p_{\beta-1}^{\infty} \mid z$ is satisfied since $a_{i} \in[\mathbb{Z}]_{P}$ and $p_{\beta-1}^{\infty} \mid z_{i}$ for each $z_{i}$. 
To generate the witness $w$, for each $i$, let $w_{i}$ be the root $r_{1}$ of the $\left[\mathcal{G}\left(\Sigma_{f_{\beta-1}(1)}^{0}\right)\right]_{u_{\beta-1,1}}$ component of $\mathcal{G}_{i}$. The conditions $u_{\beta-1,1}^{\infty} \mid a_{i} w_{i}$ and $v_{\beta-1,0}^{\infty} \mid\left(a_{i} z_{i}+a_{i} w_{i}\right)$ are satisfied since $z_{i}=r_{0}$ and $w_{i}=r_{1}$ in $\mathcal{G}_{i}$. It follows that $\mathcal{G} \mid=A_{\beta-1}(z)$ with witness $w=\sum a_{i} w_{i}$.

To see that $\mathcal{G}=\Psi_{\beta-1}(z)$, we reason as follows. Fix $m \in \omega$. We show how to pick the witnessing elements $g_{0}, \ldots, g_{m}$ for the $m$-th conjunct. For each $z_{i}$, pick a sequence of elements $g_{i, 0}, g_{i, 1}, \ldots, g_{i, m}$ in $\mathcal{G}_{i}$ by setting $g_{i, 0}=z_{i}$ (which is the $r_{0}$ root in $\mathcal{G}_{i}$ ) and $g_{i, k}=r_{k}$ (the root of the $\left[\mathcal{G}\left(\Sigma_{f_{\beta-1}(k)}^{0}\right)\right]_{u_{\beta-1, k}}$ component of $\mathcal{G}_{i}$ ) for $0<k \leq m$. Since $a_{i} \in[\mathbb{Z}]_{P}$ and each $\mathcal{G}_{i}$ is $P$-closed, we have (from the structure of $\left.\mathcal{G}\left(\Pi_{\beta-1}^{0}\right)\right)$ that $u_{\beta-1, k}^{\infty} \mid a_{i} g_{i, k}$ for $k \leq m$ and $v_{\beta-1, k}^{\infty} \mid\left(a_{i} g_{i, k}+a_{i} g_{i, k+1}\right)$ for $k<m$.

For each $0 \leq k \leq m$, let $g_{k}=\sum_{i} a_{i} g_{i, k}$. By the divisibility conditions above, we have $u_{\beta-1, k}^{\infty} \mid g_{k}$ for $k \leq m$ and $v_{\beta-1, k}^{\infty} \mid\left(g_{k}+g_{k+1}\right)$ for $k<m$. Furthermore, $g_{0}=z$. Therefore, it only remains to show that $\Phi_{f_{\beta-1}(m)}^{u_{\beta-1, m}}\left(g_{m}\right)$. We already have $u_{\beta-1, m}^{\infty} \mid g_{m}$. Since $g_{m}=\sum_{i} a_{i} g_{i, m}$ where $a_{i} \in[\mathbb{Z}]_{P}$ and $g_{i, m}$ is the root of a $\left[\mathcal{G}\left(\Sigma_{f_{\beta-1}(m)}^{0}\right)\right]_{u_{\beta-1, m}}$ component, it follows from Part (3)(b) or (4)(b), depending on the form of $f_{\beta-1}(m)$, that $\mathcal{G}$ satisfies $\Phi_{f_{\beta-1}(m)}^{u_{\beta-1, m}}\left(g_{m}\right)$ and hence $\mathcal{G} \models \Psi_{\beta-1}(z)$.

(4) As $f_{\beta-1}(k)$ is an odd ordinal and $f_{\beta-1}(k)<\beta-1$ for all $k \in \omega$, the proof of Part (4) is essentially the same as the proof of Part (3) with the appropriate notational changes to reflect that $\beta-1$ is a limit ordinal.

Lemma 4.17. Let $\beta=\delta+2 \ell+1 \geq 3$. Then for $\mathcal{G}=\bigoplus_{n \in \omega} \mathcal{G}_{n}$, where $\mathcal{G}_{n}$ is either $\left[\mathcal{G}\left(\Sigma_{\beta}^{0}\right)\right]_{d_{n}}$ or $\left[\mathcal{G}\left(\Pi_{\beta}^{0}\right)\right]_{d_{n}}$, the following holds:

$$
\mathcal{G}=\left[(\exists x) \Phi_{\beta}(x)\right]^{d_{n}} \quad \text { if and only if } \quad \mathcal{G}_{n} \cong\left[\mathcal{G}\left(\Sigma_{\beta}^{0}\right)\right]_{d_{n}} .
$$

Proof. Since $\mathcal{G}_{n}$ is the subgroup of elements of $\mathcal{G}$ which are infinitely divisible by $d_{n}$, we have by Lemma 4.6

$$
\mathcal{G}=\left[(\exists x) \Phi_{\beta}(x)\right]^{d_{n}} \Leftrightarrow \mathcal{G}_{n}=(\exists x) \Phi_{\beta}(x)
$$

Therefore, it suffices to show that $\mathcal{G}\left(\Sigma_{\beta}^{0}\right) \models(\exists x) \Phi_{\beta}(x)$ and $\mathcal{G}\left(\Pi_{\beta}^{0}\right) \not \models(\exists x) \Phi_{\beta}(x)$.

First, we show that $\mathcal{G}\left(\Sigma_{\beta}^{0}\right) \models \Phi_{\beta}(r)$ where $r$ is the root of $\mathcal{G}\left(\Sigma_{\beta}^{0}\right)$. That is, we show that $\mathcal{G}\left(\Sigma_{\beta}^{0}\right)$ satisfies

$$
p_{\beta}^{\infty} \mid r \wedge(\exists y)\left[q_{\beta}^{\infty} \mid(r+y) \wedge A_{\beta-1}(y) \wedge \Psi_{\beta-1}(y)\right]
$$

Since $r$ is the root of $\mathcal{G}\left(\Sigma_{\beta}^{0}\right)$, we immediately obtain $p_{\beta}^{\infty} \mid r$. We claim that the root $r_{k}$ of a $\mathcal{G}\left(\Pi_{\beta-1}^{0}\right)$ component works for the choice of $y$ in $\Phi_{\beta}$. From the definition of $\mathcal{G}\left(\Sigma_{\beta}^{0}\right)$, we have $q_{\beta}^{\infty} \mid\left(r+r_{k}\right)$ and by Lemma $4.13(2)(\mathrm{b})$, we have that $\mathcal{G}\left(\Sigma_{\beta}^{0}\right)$ satisfies both $A_{\beta-1}\left(r_{k}\right)$ and $\Psi_{\beta-1}\left(r_{k}\right)$ as required.

Second, assume for a contradiction that $\mathcal{G}\left(\Pi_{\beta}^{0}\right) \models(\exists x) \Phi_{\beta}(x)$ and fix the witness $x$. The condition $p_{\beta}^{\infty} \mid x$ implies that $x$ is a multiple of the root of $\mathcal{G}\left(\Pi_{\beta}^{0}\right)$. Fix the witness $y$ such that $q_{\beta}^{\infty} \mid(x+y) \wedge A_{\beta-1}(y) \wedge \Psi_{\beta-1}(y)$. By Lemma 4.13(2)(a), the condition $A_{\beta-1}(y) \wedge \Psi_{\beta-1}(y)$ implies that $y$ is a sum of multiples of the roots of $\mathcal{G}\left(\Pi_{\beta-1}^{0}\right)$ components. However, $\mathcal{G}\left(\Pi_{\beta}^{0}\right)$ has no such components, giving the desired contradiction. 
We continue by constructing sentences connected semantically to $\mathcal{H}\left(\Sigma_{\beta}^{0}\right)$ and $\mathcal{H}\left(\Pi_{\beta}^{0}\right)$. We first give lemmas similar to Lemma 4.13 for the groups $\mathcal{H}\left(\Sigma_{\beta}^{0}\right)$ and $\mathcal{H}\left(\Pi_{\beta}^{0}\right)$.

Lemma 4.18. Let $\beta=\delta+2 l+2 \geq 4$ and let $\mathcal{H} \cong \mathcal{H}\left(\Pi_{\beta}^{0}\right)$. Let $y \in \mathcal{H}$ be a sum $y=\sum b_{j} y_{j}$ where each $b_{j} \in \mathbb{Z}$ and each $y_{j}$ is the root of a (distinct) $\mathcal{G}\left(\Sigma_{\beta-1}^{0}\right)$ component of $\mathcal{H}$. Then $\mathcal{H} \models \Phi_{\beta-1}(y)$.

Proof. We need to show that $\mathcal{H}$ satisfies

$$
p_{\beta-1}^{\infty} \mid y \wedge(\exists w)\left[q_{\beta-1}^{\infty} \mid(y+w) \wedge A_{\beta-2}(w) \wedge \Psi_{\beta-2}(w)\right]
$$

By the structure of $\mathcal{G}\left(\Sigma_{\beta-1}^{0}\right), p_{\beta-1}^{\infty} \mid y_{j}$ for each $j$. Since $b_{j} \in \mathbb{Z}$, we have $p_{\beta-1}^{\infty} \mid b_{j} y_{j}$ and hence $p_{\beta-1}^{\infty} \mid y$. For each $j$, let $w_{j}$ be the root of a $\mathcal{G}\left(\Pi_{\beta-2}^{0}\right)$ component within the $\mathcal{G}\left(\Sigma_{\beta-1}^{0}\right)$ component with root $y_{j}$ and let $w=\sum b_{j} w_{j}$. It follows from the structure of $\mathcal{G}\left(\Sigma_{\beta-1}^{0}\right)$ that $q_{\beta-1}^{\infty} \mid(y+w)$. Therefore, it remains to show that $\mathcal{H}$ satisfies $A_{\beta-2}(w) \wedge \Psi_{\beta-2}(w)$.

The group $\mathcal{H}$ is built by taking a direct sum of the groups $[\mathbb{Z}]_{p_{\beta}}$ (with root $r$ ) and $\bigoplus_{k \in \omega} \mathcal{G}\left(\Sigma_{\beta-1}^{0}\right)$ (with roots $r_{k}$ ) and then adding extra elements (from the divisible closure of this sum) to witness $q_{\beta}^{\infty} \mid\left(r+r_{k}\right)$. Since $w=\sum b_{j} w_{j}$ with each $b_{j} \in \mathbb{Z}$, we can view $w$ as an element of the group $\bigoplus_{k \in \omega} \mathcal{G}\left(\Sigma_{\beta-1}^{0}\right)$ in this construction of $\mathcal{H}$. By Lemma 4.13(2)(b) applied to $w$ as an element of $\bigoplus_{k \in \omega} \mathcal{G}\left(\Sigma_{\beta-1}^{0}\right)$, we have that $\bigoplus_{k \in \omega} \mathcal{G}\left(\Sigma_{\beta-1}^{0}\right)$ satisfies $A_{\beta-2}(w) \wedge \Psi_{\beta-2}(w)$. Since $\bigoplus_{k \in \omega} \mathcal{G}\left(\Sigma_{\beta-1}^{0}\right)$ is a subgroup of $\mathcal{H}$, Lemma 4.12 implies that $\mathcal{H}$ satisfies $A_{\beta-2}(w) \wedge \Psi_{\beta-2}(w)$ as required.

Lemma 4.19. Let $\beta=\delta+2 l+2 \geq 4$ and let $\mathcal{H} \cong \mathcal{H}\left(\Sigma_{\beta}^{0}\right)$. Let $r$ be the root of a $\mathcal{G}\left(\Pi_{\beta-1}^{0}\right)$ component of $\mathcal{H}$. Then $\mathcal{H} \forall \Phi_{\beta-1}(r)$.

Proof. By the structure of $\mathcal{G}\left(\Pi_{\beta-1}^{0}\right)$, we have that $p_{\beta-1}^{\infty} \mid r$, so we need to show that there is no $w \in \mathcal{H}$ such that $\mathcal{H}$ satisfies

$$
q_{\beta-1}^{\infty} \mid(r+w) \wedge A_{\beta-2}(w) \wedge \Psi_{\beta-2}(w)
$$

For a contradiction, fix such an element $w \in \mathcal{H}$. To simplify dealing with $q_{\beta}$ divisibility in $\mathcal{H}$, we work in the expanded group $[\mathcal{H}]_{q_{\beta}}$ and note that if $w$ satisfies this formula in $\mathcal{H}$, then by Lemma 4.12 , it also satisfies the formula in $[\mathcal{H}]_{q_{\beta}}$.

The group $[\mathcal{H}]_{q_{\beta}}$ decomposes as a direct sum

$$
[\mathbb{Z}]_{p_{\beta}, q_{\beta}} \oplus \bigoplus_{i \in \omega}\left[\mathcal{C}_{i}\right]_{q_{\beta}}
$$

where each $\mathcal{C}_{i}$ is isomorphic to $\mathcal{G}\left(\Sigma_{\beta-1}^{0}\right)$ or $\mathcal{G}\left(\Pi_{\beta-1}^{0}\right)$. The divisibility condition $p_{\beta-2}^{\infty} \mid w$ (from the fact that $\mathcal{H} \models A_{\beta-2}(w)$ ) implies that $w=\sum a_{i} w_{i}$ where each $a_{i} \in \mathbb{Q}$ and each $w_{i}$ is the root of a $\mathcal{G}\left(\Sigma_{\beta-2}^{0}(m)\right)$ or $\mathcal{G}\left(\Pi_{\beta-2}^{0}\right)$ component. Therefore, as an element of $[\mathcal{H}]_{q_{\beta}}, w \in \bigoplus_{i \in \omega}\left[\mathcal{C}_{i}\right]_{q_{\beta}}$. In addition, the condition $q_{\beta-1}^{\infty} \mid(r+w)$ implies that at least one $w_{i}$ is the root of a $\mathcal{G}\left(\Sigma_{\beta-2}^{0}(m)\right)$ subcomponent of the $\mathcal{G}\left(\Pi_{\beta-1}^{0}\right)$ component with root $r$ (as this component has no $\mathcal{G}\left(\Pi_{\beta-2}^{0}\right)$ subcomponents).

Assume for a moment that $\bigoplus_{i \in \omega}\left[\mathcal{C}_{i}\right]_{q_{\beta}}$ satisfies $A_{\beta-2}(w) \wedge \Psi_{\beta-2}(w)$. Under this assumption, Lemma 4.13(2)(a) implies that $w$ is a sum of roots of $\mathcal{G}\left(\Pi_{\beta-2}^{0}\right)$ components, contradicting the fact that at least one $w_{i}$ is the root of a $\mathcal{G}\left(\Sigma_{\beta-2}^{0}(m)\right)$ component. Therefore, to complete our proof, it suffices to show that $\bigoplus_{i \in \omega}\left[\mathcal{C}_{i}\right]_{q_{\beta}}$ satisfies $A_{\beta-2}(w) \wedge \Psi_{\beta-2}(w)$. 
To show $\bigoplus_{i \in \omega}\left[\mathcal{C}_{i}\right]_{q_{\beta}}$ satisfies $A_{\beta-2}(w) \wedge \Psi_{\beta-2}(w)$, we use the fact that $\bigoplus_{i \in \omega}\left[\mathcal{C}_{i}\right]_{q_{\beta}}$ is a pure subgroup of $[\mathcal{H}]_{q_{\beta}}$ (since it is a direct summand) along with the following observation. Because $[\mathcal{H}]_{q_{\beta}}$ is a direct sum, any element $z \in[\mathcal{H}]_{q_{\beta}}$ can be written (uniquely) in the form $z=z_{0}+z_{1}$ where $z_{0} \in[\mathbb{Z}]_{p_{\beta}, q_{\beta}}$ and $z_{1} \in \bigoplus_{i \in \omega}\left[\mathcal{C}_{i}\right]_{q_{\beta}}$. If $\rho$ is a prime and $\rho^{\infty} \mid z$, then $\rho^{\infty} \mid z_{0}$ and $\rho^{\infty} \mid z_{1}$. Therefore, if $\rho^{\infty} \mid z$ and $\rho$ is not $p_{\beta}$ or $q_{\beta}$, we can conclude that $z \in \bigoplus_{i \in \omega}\left[\mathcal{C}_{i}\right]_{q_{\beta}}$.

Using this observation, we show that the following property holds for all $\gamma$ such that $2 \leq \gamma \leq \beta-2$. Let $\varphi(x)$ be either $A_{\gamma}(x)$ or $\Psi_{\gamma}(x)$ (if $\gamma$ is even) or $\Phi_{\gamma}(x)$ (if $\gamma$ is odd) and let $\rho$ be any prime number. For any $x \in \bigoplus_{i \in \omega}\left[\mathcal{C}_{i}\right]_{q_{\beta}}$, we have

$$
\begin{gathered}
{[\mathcal{H}]_{q_{\beta}}=\varphi(x) \Rightarrow \bigoplus_{i \in \omega}\left[\mathcal{C}_{i}\right]_{q_{\beta}} \models \varphi(x) \text { and }} \\
{[\mathcal{H}]_{q_{\beta}}=\varphi^{\rho}(x) \Rightarrow \bigoplus_{i \in \omega}\left[\mathcal{C}_{i}\right]_{q_{\beta}}=\varphi^{\rho}(x)}
\end{gathered}
$$

Notice that establishing this property finishes our proof as $w \in \bigoplus_{i \in \omega}\left[\mathcal{C}_{i}\right]_{q_{\beta}}$ and $[\mathcal{H}]_{q_{\beta}}=A_{\beta-2}(w) \wedge \Psi_{\beta-2}(w)$, so by the property $\bigoplus_{i \in \omega}\left[\mathcal{C}_{i}\right]_{q_{\beta}} \models A_{\beta-2}(w) \wedge \Psi_{\beta-2}(w)$.

First, consider the case when $\varphi(x)$ is $A_{\gamma}(x)$ and assume $[\mathcal{H}]_{q_{\beta}} \models A_{\gamma}(x)$. In this case, the existential witness $y$ in $A_{\gamma}(x)$ is infinitely divisible by $u_{\gamma, 1}$. By the observation above, $y \in \bigoplus_{i \in \omega}\left[\mathcal{C}_{i}\right]_{q_{\beta}}$. Since $\bigoplus_{i \in \omega}\left[\mathcal{C}_{i}\right]_{q_{\beta}}$ is a pure subgroup containing $x$ and $y, \bigoplus_{i \in \omega}\left[\mathcal{C}_{i}\right]_{q_{\beta}}$ satisfies $A_{\gamma}(x)$. The same proof works for $A_{\beta}^{\rho}(x)$.

Second, consider the cases when $\varphi(x)$ is $\Phi_{\gamma}(x)$ (for odd $\gamma$ ), $\Psi_{\gamma}(x)$ (for even $\gamma$ ) or a prime relativization of one of these formulas. We proceed by induction on $\gamma$ and note that in each case the proof for the relativized formula is identical to the proof for the unrelativized formula. In each case, we assume $x \in \bigoplus_{i \in \omega}\left[\mathcal{C}_{i}\right]_{q_{\beta}}$ and $[\mathcal{H}]_{q_{\beta}}=\varphi(x)$.

The first base case is when $\beta=2$. Since $\Psi_{2}(x)$ is $p_{1}^{\infty} \mid x, x \in \bigoplus_{i \in \omega}\left[\mathcal{C}_{i}\right]_{q_{\beta}}$ and $\bigoplus_{i \in \omega}\left[\mathcal{C}_{i}\right]_{q_{\beta}}$ is a pure subgroup, we have $\bigoplus_{i \in \omega}\left[\mathcal{C}_{i}\right]_{q_{\beta}} \models \Psi_{2}(x)$.

The second base case is $\beta=3$. The existential witness $y$ in the formula $\Phi_{3}(x)$ satisfies $p_{1}^{\infty} \mid y\left(\right.$ from $\left.\Psi_{2}(y)\right)$. By the observation, $y \in \bigoplus_{i \in \omega}\left[\mathcal{C}_{i}\right]_{q_{\beta}}$. Since $\bigoplus_{i \in \omega}\left[\mathcal{C}_{i}\right]_{q_{\beta}}$ is a pure subgroup containing $x$ and $y, \bigoplus_{i \in \omega}\left[\mathcal{C}_{i}\right]_{q_{\beta}}=\Phi_{3}(x)$.

For the inductive cases, suppose $\gamma$ is even and $4 \leq \gamma \leq \beta-2$. Consider the $m$-th conjunct of $\Psi_{\gamma}(x)$. The witnesses $x_{0}, \ldots, x_{m}$ satisfy $u_{\gamma, k}^{\infty} \mid x_{k}$ and thus by the observation are in $\bigoplus_{i \in \omega}\left[\mathcal{C}_{i}\right]_{q_{\beta}}$. Since $\bigoplus_{i \in \omega}\left[\mathcal{C}_{i}\right]_{q_{\beta}}$ is a pure subgroup and it satisfies $\Phi_{f_{\gamma}(m)}^{u_{\gamma, m}}\left(x_{m}\right)$ by the inductive hypothsis, we have that $\bigoplus_{i \in \omega}\left[\mathcal{C}_{i}\right]_{q_{\beta}} \models \Psi_{\gamma}(x)$.

If $\gamma$ is odd and $4<\gamma \leq \beta-2$, then the existential witness $y$ in $\Phi_{\gamma}(x)$ satisfies $p_{\gamma-1}^{\infty} \mid y$ (from $\left.A_{\gamma-1}(y)\right)$. Thus, by the observation, $y \in \bigoplus_{i \in \omega}\left[\mathcal{C}_{i}\right]_{q_{\beta}}$. Since $\bigoplus_{i \in \omega}\left[\mathcal{C}_{i}\right]_{q_{\beta}}$ is a pure subgroup and satisfies $A_{\gamma-1}(y)$ and $\Psi_{\gamma-1}(y)$ by the inductive hypothesis, we have $\bigoplus_{i \in \omega}\left[\mathcal{C}_{i}\right]_{q_{\beta}} \models \Phi_{\gamma}(x)$.

Definition 4.20. If $\beta \geq 4$ is not a limit ordinal, define $B_{\beta}(x)$ to be the formula

$$
B_{\beta}(x):=p_{\beta}^{\infty} \mid x \wedge(\exists w)\left[p_{\beta-1}^{\infty}\left|w \wedge q_{\beta}^{\infty}\right|(x+w)\right] .
$$

Definition 4.21. If $\beta=\delta+2 \ell+2 \geq 4$, define $\Theta_{\beta}$ to be the formula

$$
\Theta_{\beta}(x):=(\forall y)\left[\left(B_{\beta}(x) \wedge B_{\beta-1}(y) \wedge q_{\beta}^{\infty} \mid(x+y)\right) \rightarrow \Phi_{\beta-1}(y)\right] .
$$

Lemma 4.22. The complexity of $B_{\beta}(x)$ is $\Sigma_{3}^{c}$ (independent of $\beta$ ).

$$
\text { If } \beta=\delta+2 \ell+2 \geq 4 \text {, then } \Theta_{\beta}(x) \in \Pi_{\beta}^{c} \text {. }
$$


Proof. These statements follow immediately from $p^{\infty} \mid x$ being $\Pi_{2}^{c}$ and Lemma 4.10.

Lemma 4.23. Let $\beta=\delta+2 \ell+2 \geq 4$. Let $x \in \mathcal{H}\left(\Pi_{\beta}^{0}\right)$ satisfy $B_{\beta}(x)$ with fixed witness $w$. Then $x=$ ar where $a \in \mathbb{Z}$ and $r$ is the root of $\mathcal{H}\left(\Pi_{\beta}^{0}\right)$ and $w=\sum b_{j} w_{j}$ where $b_{j} \in[\mathbb{Z}]_{p_{\beta-1}, q_{\beta}}$ and each $w_{j}$ is the root of a $\mathcal{G}\left(\Sigma_{\beta-1}^{0}\right)$ component of $\mathcal{H}\left(\Pi_{\beta}^{0}\right)$.

Proof. Since $p_{\beta}^{\infty} \mid x, x$ must have the form $x=a r$ where $a \in \mathbb{Q}$ and $r$ is the root of $\mathcal{H}\left(\Pi_{\beta}^{0}\right)$. Since $p_{\beta-1}^{\infty} \mid w, w$ must have the form $w=\sum b_{j} w_{j}$ where $b_{j} \in \mathbb{Q}$ and $w_{j}$ is the root of a $\mathcal{G}\left(\Sigma_{\beta-1}^{0}\right)$ component of $\mathcal{H}\left(\Pi_{\beta}^{0}\right)$.

Let $\mathcal{B}=\operatorname{Span}_{\mathcal{H}\left(\Pi_{\beta}^{0}\right)}(X)$ where $X$ contains the root of $\mathcal{H}\left(\Pi_{\beta}^{0}\right)$ and the roots of the $\mathcal{G}\left(\Sigma_{\beta-1}^{0}\right)$ components of $\mathcal{H}\left(\Pi_{\beta}^{0}\right)$. Then $x, w \in \mathcal{B}, \mathcal{B}$ is a pure subgroup of $\mathcal{H}\left(\Pi_{\beta}^{0}\right)$ and $\mathcal{B}$ is isomorphic to

$$
\left\langle[\mathbb{Z}]_{p_{\beta}} \oplus \oplus_{k \in \omega}[\mathbb{Z}]_{p_{\beta-1}} ; q_{\beta}^{-t}\left(r+r_{k}\right): k, t \in \omega\right\rangle
$$

Since $\mathcal{B}$ satisfies $p_{\beta}^{\infty}\left|x, p_{\beta-1}^{\infty}\right| w$ and $q_{\beta}^{\infty} \mid(x+w)$, we can apply Lemma 4.4(5) (with $P=\emptyset)$ to conclude that $a \in \mathbb{Z}$ and each $b_{j} \in[\mathbb{Z}]_{p_{\beta-1}, q_{\beta}}$.

Lemma 4.24. Let $\beta=\delta+2 \ell+2 \geq 4$. If $x, y \in \mathcal{H}\left(\Pi_{\beta}^{0}\right)$ satisfy

$$
B_{\beta}(x) \wedge B_{\beta-1}(y) \wedge q_{\beta}^{\infty} \mid(x+y)
$$

then $x=$ ar where $a \in \mathbb{Z}$ and $r$ is the root of $\mathcal{H}\left(\Pi_{\beta}^{0}\right)$ and $y=\sum b_{j} y_{j}$ where each $b_{j} \in \mathbb{Z}$ and $y_{j}$ is the root of a $\mathcal{G}\left(\Sigma_{\beta-1}^{0}\right)$ component of $\mathcal{H}\left(\Pi_{\beta}^{0}\right)$.

Proof. By the previous lemma, the fact that $B_{\beta}(x)$ holds implies $x=$ ar with $a \in \mathbb{Z}$ and $r$ the root of $\mathcal{H}\left(\Pi_{\beta}^{0}\right)$. Since $B_{\beta-1}(y)$ implies $p_{\beta-1}^{\infty} \mid y$ and since $q_{\beta}^{\infty} \mid(x+y)$, the element $y$ works as a witness $w$ in the formula $B_{\beta}(x)$ for our fixed element $x$. Therefore, by the previous lemma $y=\sum b_{j} y_{j}$ where $b_{j} \in[\mathbb{Z}]_{p_{\beta-1}, q_{\beta}}$ and $y_{j}$ is the root of a $\mathcal{G}\left(\Sigma_{\beta-1}^{0}\right)$ component. It remains to show the stronger conclusion that $b_{j} \in \mathbb{Z}$.

Fix the witness $w$ for $B_{\beta-1}(y)$. Since $p_{\beta-2}^{\infty} \mid w, w$ must have the form $w=\sum c_{i} w_{i}$ where $c_{i} \in \mathbb{Q}$ and $w_{i}$ is the root of a $\mathcal{G}\left(\Sigma_{\beta-2}^{0}(m)\right)$ component inside a $\mathcal{G}\left(\Sigma_{\beta-1}^{0}\right)$ component of $\mathcal{H}\left(\Pi_{\beta}^{0}\right)$. Therefore $y, w \in \mathcal{B}$ where $\mathcal{B}=\operatorname{Span}_{\mathcal{H}\left(\Pi_{\beta}^{0}\right)}(X)$ where $X$ contains the roots of the $\mathcal{G}\left(\Sigma_{\beta-1}^{0}\right)$ components and the roots of their $\mathcal{G}\left(\Sigma_{\beta-2}^{0}(m)\right)$ subcomponents.

To determine the isomorphism type of $\mathcal{B}$, we consider which primes infinitely divide the roots of such components. The root of a $\mathcal{G}\left(\Sigma_{\beta-1}^{0}\right)$ component is infinitely divisible by $p_{\beta-1}$. The roots of $\mathcal{G}\left(\Sigma_{\beta-2}^{0}(m)\right)$ components are infinitely divisible by $p_{\beta-2}$ and $u_{\beta-2,0}$ from the definition of $\mathcal{G}\left(\Sigma_{\beta-2}^{0}(m)\right)$. Each of these roots is also the root of a $\mathcal{G}\left(\Sigma_{f_{\beta-2}(0)}^{0}\right)$ component (inside $\mathcal{G}\left(\Sigma_{\beta-2}^{0}(m)\right)$ ) and hence is also infinite divisible by $p_{f_{\beta-2}(0)}$. However, the recursion stops at this point since the root of $\mathcal{G}\left(\Sigma_{f_{\beta-2}(0)}^{0}\right)$ is the element 1 in a copy of $[\mathbb{Z}]_{p_{f_{\beta-2}(0)}}$. Therefore, $\mathcal{B}$ is isomorphic to

$$
\left[\left\langle\bigoplus_{i \in \omega}[\mathbb{Z}]_{F_{1}} \oplus \bigoplus_{i, j \in \omega}[\mathbb{Z}]_{F_{2}} ; \frac{s_{i}+t_{i, j}}{q_{\beta-2}^{l}}: i, j, l \in \omega\right\rangle\right]
$$

where $F_{1}=\left\{p_{\beta-1}\right\}, F_{2}=\left\{p_{\beta-2}, u_{\beta-2,0}, p_{f_{\beta-2}(0)}\right\}$, the $s_{i}$ elements generate the copies of $[\mathbb{Z}]_{F_{1}}$ (representing the roots of the $\mathcal{G}\left(\Sigma_{\beta-1}^{0}\right)$ components) and the $t_{i, j}$ 
elements generate the copies of $[\mathbb{Z}]_{F_{2}}$ (representing the roots of the $\mathcal{G}\left(\Sigma_{\beta-2}^{0}(m)\right)$ subcomponents of the $\mathcal{G}\left(\Sigma_{\beta-1}^{0}\right)$ component with root $\left.s_{i}\right)$. Since $y, w \in \mathcal{B}$ and $\mathcal{B}$ is a pure subgroup of $\mathcal{H}\left(\Pi_{\beta}^{0}\right)$ satisfying $p_{\beta-1}^{\infty}\left|y, p_{\beta-2}^{\infty}\right| w$ and $q_{\beta-1}^{\infty} \mid(y+w)$, we can conclude from Lemma 4.4(5) (with $P=\emptyset$ ) that the coefficients in the sum $y=\sum b_{j} y_{j}$ come from $\mathbb{Z}$.

Lemma 4.25. Let $\beta=\delta+2 \ell+2 \geq 4$. Then for $\mathcal{H}=\bigoplus_{n \in \omega} \mathcal{H}_{n}$, where $\mathcal{H}_{n}$ is either $\left[\mathcal{H}\left(\Sigma_{\beta}^{0}\right)\right]_{d_{n}}$ or $\left[\mathcal{H}\left(\Pi_{\beta}^{0}\right)\right]_{d_{n}}$, the following holds:

$$
\mathcal{H} \models\left[(\forall x) \Theta_{\beta}(x)\right]^{d_{n}} \quad \text { if and only if } \quad \mathcal{H}_{n} \cong\left[\mathcal{H}\left(\Pi_{\beta}^{0}\right)\right]_{d_{n}} .
$$

Proof. Since $\mathcal{H}_{n}$ is the subgroup of elements of $\mathcal{H}$ which are infinitely divisible by $d_{n}$, we have

$$
\mathcal{H}=\left[(\forall x) \Theta_{\beta}(x)\right]^{d_{n}} \Leftrightarrow \mathcal{H}_{n}=(\forall x) \Theta_{\beta}(x)
$$

Therefore, it suffices to show that $\mathcal{H}\left(\Pi_{\beta}^{0}\right) \models(\forall x) \Theta_{\beta}(x)$ and $\mathcal{H}\left(\Sigma_{\beta}^{0}\right) \not \models(\forall x) \Theta_{\beta}(x)$.

First, we show that $\mathcal{H}\left(\Pi_{\beta}^{0}\right) \models(\forall x) \Theta_{\beta}(x)$. Fix elements $x, y \in \mathcal{H}\left(\Pi_{\beta}^{0}\right)$ satisfying $B_{\beta}(x) \wedge B_{\beta-1}(y) \wedge q_{\beta}^{\infty} \mid(x+y)$. By Lemma 4.24, $y=\sum b_{j} y_{j}$ where each $b_{j} \in \mathbb{Z}$ and $y_{j}$ is the root of a $\mathcal{G}\left(\Sigma_{\beta-1}^{0}\right)$ component. By Lemma 4.18, $y$ satisfies $\Phi_{\beta-1}(y)$ as required.

Second, we show that $\mathcal{H}\left(\Sigma_{\beta}^{0}\right) \not \models(\forall x) \Theta_{\beta}(x)$ by proving that $\mathcal{H}\left(\Sigma_{\beta}^{0}\right) \not \models \Theta_{\beta}(r)$ where $r$ is the root of $\mathcal{H}\left(\Sigma_{\beta}^{0}\right)$. Let $y$ be the root of a $\mathcal{G}\left(\Pi_{\beta-1}^{0}\right)$ component of $\mathcal{H}\left(\Sigma_{\beta}^{0}\right)$. It is immediate that $\mathcal{H}\left(\Sigma_{\beta}^{0}\right) \models B_{\beta}(r) \wedge B_{\beta-1}(y) \wedge q_{\beta}^{\infty} \mid(r+y)$. However, by Lemma $4.19, \mathcal{H}\left(\Sigma_{\beta}^{0}\right)$ does not satisfy $\Phi_{\beta-1}(y)$.

Definition 4.26. Define sentences $\Upsilon_{n}$ for $n \in \omega$ as follows.

- If $\alpha=\delta+2 \ell+1 \geq 3$, let $\Upsilon_{n}:=\left[(\exists x) \Phi_{\alpha}(x)\right]^{d_{n}}$.

- If $\alpha=\delta+2 \ell+2 \geq 4$, let $\Upsilon_{n}:=\neg\left[(\forall x) \Theta_{\alpha}(x)\right]^{d_{n}}$.

Proof of Lemma 4.1. By Lemma 4.17 and Lemma 4.25, the sentences $\Upsilon_{n}$ have the desired semantic properties. As a consequence of Lemma 4.10 and Lemma 4.22, the formulas $\Upsilon_{n}$ have the desired quantifier complexity. Moreover, all the (sub)formulas are computable with all possible uniformity, so $\Upsilon_{n}$ is uniformly computably $\Sigma_{\alpha}^{c}$.

4.2. Proof of Lemma 4.2. The construction of an $X$-computable copy of $\mathcal{G}_{S}$ if $S \in \Sigma_{\alpha}^{0}(X)$ is also done by recursion. We treat only the case when $X=\emptyset$, the more general case following by relativization.

Lemma 4.27. For every even ordinal $\beta=\delta>0$ or $\beta=\delta+2 \ell+2 \geq 2$ and $\Sigma_{\beta}^{0}$ set $S$, there is a uniformly computable sequence $\left\{\mathcal{G}_{n}\right\}_{n \in \omega}$ of rooted torsion-free abelian groups such that $\mathcal{G}_{n} \cong \mathcal{G}\left(\Sigma_{\beta}^{0}(m)\right)$ for some $m \in \omega$ if $n \in S$ and $\mathcal{G}_{n} \cong \mathcal{G}\left(\Pi_{\beta}^{0}\right)$ if $n \notin S$.

For every odd ordinal $\beta=\delta+2 \ell+1 \geq 3$ and $\Sigma_{\beta}^{0}$ set $S$, there is a uniformly computable sequence $\left\{\mathcal{G}_{n}\right\}_{n \in \omega}$ of rooted torsion-free abelian groups such that $\mathcal{G}_{n} \cong$ $\mathcal{G}\left(\Sigma_{\beta}^{0}\right)$ if $n \in S$ and $\mathcal{G}_{n} \cong \mathcal{G}\left(\Pi_{\beta}^{0}\right)$ if $n \notin S$.

Moreover the passage from an index for the set $S$ to an index for the sequence is effective.

Proof. The proof is done by induction on $\beta$. We treat the cases $\beta=2, \beta=$ $\delta+2 \ell+2 \geq 4, \beta=\delta+2 \ell+1 \geq 3$, and $\beta=\delta>0$ separately. In all cases, we fix a predicate $(\exists s)[R(n, s)]$ describing membership of $n$ in $S$, where $R(n, s)$ is $\Pi_{f_{\beta}(k)}^{0}$ for 
some $k$. Without loss of generality, we suppose $R\left(n, s_{0}\right)$ implies $\left(\forall s \geq s_{0}\right)[R(n, s)]$. Indeed, we suppose this property of all existential subpredicates.

For $\beta=2$, it suffices to start with the group $\mathbb{Z}$ with root $r_{n}=1$ for $\mathcal{G}_{n}$. When we see $\neg R(n, s)$ for a new existential witness $s$, we introduce the element $1 / p^{s}$ into the group. It is easy to see the sequence $\left\{\mathcal{G}_{n}\right\}_{n \in \omega}$ has the desired properties.

For $\beta=\delta+2 \ell+1 \geq 3$, it suffices to start with the group $[\mathbb{Z}]_{p_{\beta}}$ with root $r_{n}=1$ for $\mathcal{G}_{n}$. For each integer $s$, we construct (via induction as $\neg R(n, s)$ is $\Sigma_{\delta+2 \ell}^{0}$ ) a rooted torsion-free abelian group $\mathcal{G}_{n, s}$ with root $r_{n, s}$ and introduce elements $\left(r_{n}+\right.$ $\left.r_{n, s}\right) / q_{\beta}^{t}$ for all $t \in \omega$. For each integer $m$, we construct infinitely many copies of $\mathcal{G}\left(\Sigma_{\beta-1}^{0}(m)\right)$ with root $r_{n, k, m}$ (where $k$ is the copy number) and introduce elements $\left(r_{n}+r_{n, k, m}\right) / q_{\beta}^{t}$ for all $t \in \omega$. Again, it is easy to see the sequence $\left\{\mathcal{G}_{n}\right\}_{n \in \omega}$ has the desired properties.

For $\beta=\delta+2 \ell+2 \geq 4$, we construct (via induction as $\neg R(n, s)$ is $\Sigma_{\delta+2 \ell+1}^{0}{ }^{7}$ ) rooted torsion-free abelian groups $\mathcal{G}_{n, s}$ with root $r_{n, s}$. Within $\mathcal{G}_{n, 0}$, we introduce elements $r_{n, 0} / p_{\beta}^{t}$ for all $t \in \omega$. Within each group $\mathcal{G}_{n, s}$, we introduce elements $x / u_{\beta, s}^{t}$ for all $t \in \omega$ and $x \in \mathcal{G}_{n, s}$. For each integer $s$, we introduce elements $\left(r_{n, s}+r_{n, s+1}\right) / v_{\beta, s}^{t}$ for all $t \in \omega$. Again, it is easy to see the sequence $\left\{\mathcal{G}_{n}\right\}_{n \in \omega}$ has the desired properties.

For $\beta=\delta$, we construct (via induction) rooted torsion-free abelian groups $\mathcal{G}_{n, s}$ with root $r_{n, s}$, where $\mathcal{G}_{n, 0} \cong \mathcal{G}\left(\Sigma_{f_{\beta}(0)}^{0}\right)$ and $\mathcal{G}_{n, s+1} \cong \mathcal{G}\left(\Pi_{f_{\beta}(s)}^{0}\right)$ if $\emptyset^{f_{\beta}(s)}$ suffices to witness $n \in S$ and $\mathcal{G}_{n, s+1} \cong \mathcal{G}\left(\Sigma_{f_{\beta}(s)}^{0}\right)$ otherwise. Within $\mathcal{G}_{n, 0}$, we introduce elements $r_{n, 0} / p_{\beta}^{t}$ for all $t \in \omega$. Within each group $\mathcal{G}_{n, s}$, we introduce elements $x / u_{\beta, s}^{t}$ for all $t \in \omega$ and $x \in \mathcal{G}_{n, s}$. For each integer $s$, we introduce elements $\left(r_{n, s}+r_{n, s+1}\right) / v_{\beta, s}^{t}$ for all $t \in \omega$. Again, it is easy to see the sequence $\left\{\mathcal{G}_{n}\right\}_{n \in \omega}$ has the desired properties.

Lemma 4.28. For every even ordinal $\beta=\delta+2 \ell+2 \geq 4$ and $\Sigma_{\beta}^{0}$ set $S$, there is a uniformly computable sequence of rooted torsion-free abelian groups $\left\{\mathcal{H}_{n}\right\}_{n \in \omega}$ such that $\mathcal{H}_{n} \cong \mathcal{H}\left(\Sigma_{\beta}^{0}\right)$ if $n \in S$ and $\mathcal{H}_{n} \cong \mathcal{H}\left(\Pi_{\beta}^{0}\right)$ if $n \notin S$.

Proof. We fix a predicate $(\exists s)[R(n, s)]$ describing membership of $n$ in $S$, where $R(n, s)$ is $\Pi_{\beta-1}^{0}$. Without loss of generality, we again suppose $R\left(n, s_{0}\right)$ implies $\left(\forall s \geq s_{0}\right)[R(n, s)]$. Indeed, we suppose this property of all existential subpredicates.

It suffices to start with the group $[\mathbb{Z}]_{p_{\beta}}$ with root $r_{n}=1$ for $\mathcal{H}_{n}$. For each integer $s$, we (via Lemma 4.27) construct a rooted torsion-free abelian group $\mathcal{G}_{n, s}$ with root $r_{n, s}$ and introduce elements $\left(r_{n}+r_{n, s}\right) / q_{\beta}^{t}$ for all $t \in \omega$. We also construct infinitely many copies of $\mathcal{G}\left(\Sigma_{\beta-1}^{0}\right)$ with root $r_{n, k}$ (where $k$ is the copy number) and introduce elements $\left(r_{n}+r_{n, k}\right) / q_{\beta}^{t}$ for all $t \in \omega$. Again, it is easy to see the sequence $\left\{\mathcal{G}_{n}\right\}_{n \in \omega}$ has the desired properties.

Proof of Lemma 4.2. Fix a $\Sigma_{\alpha}^{0}$ set $S$. From Lemma 4.27 (if $\alpha$ is odd) or Lemma 4.28 (if $\alpha$ is even), there is a uniformly computable sequence $\left\{\mathcal{G}_{n}\right\}_{n \in \omega}$ of groups given by the $\Sigma_{\alpha}^{0}$ predicate. Since it is possible to pass from the group $\mathcal{G}_{n}$ to $\left[\mathcal{G}_{n}\right]_{d_{n}}$ uniformly in an index for the group $\mathcal{G}_{n}$ and $d_{n}$, the group $\mathcal{G}_{S}$ is computable.

\footnotetext{
${ }^{7}$ More precisely, we use the $\Sigma_{\delta+2 \ell+1}^{0}$ predicate $\neg R(n, s)$ to control the construction of $\mathcal{G}_{n, s+1}$ and build $\mathcal{G}_{n, 0} \cong \mathcal{G}\left(\Sigma_{\beta-1}^{0}\right)$. This index shift is necessary as $\mathcal{G}\left(\Sigma_{\beta}^{0}(m)\right)$ has $m+1$ (rather than $m$ ) subcomponents of type $\mathcal{G}\left(\Sigma_{\beta-1}^{0}\right)$.
} 


\section{REFERENCES}

[1] C. J. Ash, C. G. Jockusch, Jr., and J. F. Knight. Jumps of orderings. Trans. Amer. Math. Soc., 319(2):573-599, 1990.

[2] C. J. Ash and J. Knight. Computable structures and the hyperarithmetical hierarchy, volume 144 of Studies in Logic and the Foundations of Mathematics. North-Holland Publishing Co., Amsterdam, 2000.

[3] Richard J. Coles, Rod G. Downey, and Theodore A. Slaman. Every set has a least jump enumeration. J. London Math. Soc. (2), 62(3):641-649, 2000.

[4] Rod Downey. Computability, definability and algebraic structures. In Proceedings of the 7th and 8th Asian Logic Conferences, pages 63-102, Singapore, 2003. Singapore Univ. Press.

[5] Rodney G. Downey. On presentations of algebraic structures. In Complexity, logic, and recursion theory, volume 187 of Lecture Notes in Pure and Appl. Math., pages 157-205. Dekker, New York, 1997.

[6] Rodney Downey and Julia F. Knight. Orderings with $\alpha$ th jump degree $\mathbf{0}^{(\alpha)}$. Proc. Amer. Math. Soc., 114(2):545-552, 1992.

[7] Rod Downey and Antonio Montalbán. The isomorphism problem for torsion-free abelian groups is analytic complete. J. Algebra, 320(6):2291-2300, 2008.

[8] Ekaterina Fokina, Julia F. Knight, C. Maher, Alexander G. Melnikov, and Sara Quinn. Classes of Ulm type, and relations between the class of rank-homogeneous trees and other classes (accepted).

[9] Harvey M. Friedman, Stephen G. Simpson and Rick L. Smith. Countable algebra and set existence axioms. Ann. Pure Appl. Logic 25:141-181, 1983.

[10] László Fuchs. Infinite abelian groups. Vol. I. Pure and Applied Mathematics, Vol. 36. Academic Press, New York, 1970.

[11] László Fuchs. Infinite abelian groups. Vol. II. Academic Press, New York, 1973. Pure and Applied Mathematics. Vol. 36-II.

[12] Greg Hjorth. The isomorphism relation on torsion free abelian groups. Fund. Math., 175(3):241-257, 2002.

[13] Carl G. Jockusch, Jr. and Robert I. Soare. Degrees of orderings not isomorphic to recursive linear orderings. Ann. Pure Appl. Logic, 52(1-2):39-64, 1991. International Symposium on Mathematical Logic and its Applications (Nagoya, 1988).

[14] Carl G. Jockusch, Jr. and Robert I. Soare. Boolean algebras, Stone spaces, and the iterated Turing jump. J. Symbolic Logic, 59(4):1121-1138, 1994.

[15] Julia F. Knight. Degrees coded in jumps of orderings. J. Symbolic Logic, 51(4):1034-1042, 1986.

[16] A. I. Mal'cev. On recursive Abelian groups. Dokl. Akad. Nauk SSSR, 146:1009-1012, 1962.

[17] Alexander G. Melnikov. Enumerations and completely decomposable torsion-free abelian groups. Theory of Comp. Sys., 45(4):897-916, 2009.

[18] Linda Jean Richter. Degrees of structures. J. Symbolic Logic, 46(4):723-731, 1981.

[19] Hartley Rogers, Jr. Theory of recursive functions and effective computability. MIT Press, Cambridge, MA, second edition, 1987.

[20] Rick L. Smith. Two theorems on autostability in p-groups. In Logic Year 1979-80, volume 859 of Lecture Notes in Math., pages 302-311. Springer-Verlag, Heidelberg, 1981.

[21] Reed Solomon. Reverse mathematics and fully ordered groups. Notre Dame J. Formal Logic, 39(2):157-189, 1998. 
Department of Mathematics and Computer Science, Assumption College, Worcester, MA 01609-1296, USA

E-mail address: brandersen@assumption.edu

Department of Mathematics, University of Connecticut, Storrs, CT 06269-3009, USA

E-mail address: asher.kach@uconn.edu

Department of Computer Science, University of Auckland, Auckland 1142, NEW ZEALAND

E-mail address: a.melnikov@cs.auckland.ac.nz

Department of Mathematics, University of Connecticut, Storrs, CT 06269-3009, USA

E-mail address: david.solomon@uconn.edu 